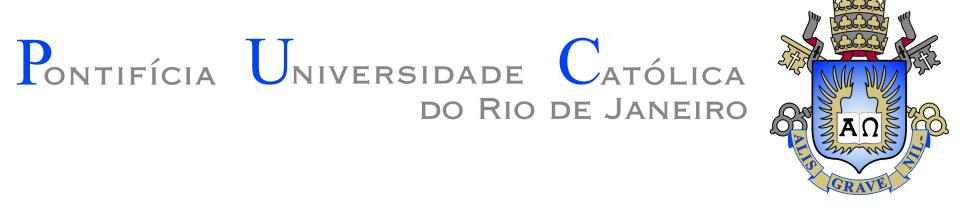

Miguel Beltran Walker Ureña

\title{
Regularity transmission by approximation methods: the Isaacs equation
}

Dissertação de Mestrado

Dissertation presented to the Programa de Pós-graduação em Matemática da PUC-Rio in partial fulfillment of the requirements for the degree of Mestre em Matemática.

Advisor: Prof. Edgard Almeida Pimentel 


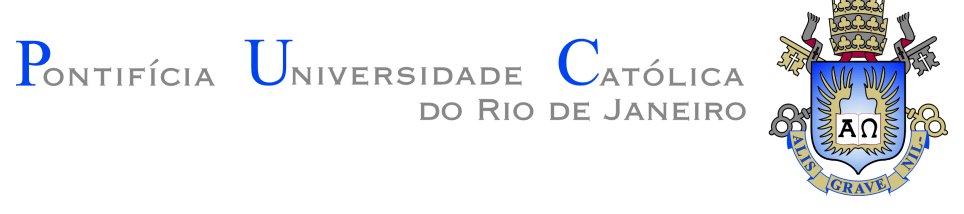

Miguel Beltran Walker Ureña

\title{
Regularity transmission by approximation methods: the Isaacs equation
}

Dissertation presented to the Programa de Pós-graduação em Matemática da PUC-Rio in partial fulfillment of the requirements for the degree of Mestre em Matemática. Approved by the Examination Committee.

\author{
Prof. Edgard Almeida Pimentel \\ Advisor \\ Departamento de Matemática - PUC-Rio
}

Prof. Boyan Slavchev Sirakov

Departamento de Matemática - PUC-Rio

Prof. Carlos Tomei

Departamento de Matemática - PUC-Rio

Prof. Jose Felipe Linares Ramírez

Instituto Nacional de Matemática Pura e Aplicada - IMPA

Prof. Juliana Honda Lopes

Departamento de Matematica - UFRJ

Prof. Mayra Soares Costa Rodrigues

Departamento de Matemática - PUC-Rio

Rio de Janeiro, September the 19th, 2019 
All rights reserved.

\section{Miguel Beltran Walker Ureña}

Has a degree in Mathematics from University of Costa Rica, Costa Rica.

Bibliographic data

Walker Ureña, Miguel Beltran

Regularity transmission by approximation methods: the Isaacs equation / Miguel Beltran Walker Ureña; advisor: Edgard Almeida Pimentel. - Rio de janeiro: PUC-Rio, Departamento de Matemática, 2019.

v., 58 f: il. color. ; $30 \mathrm{~cm}$

Dissertação (mestrado) - Pontifícia Universidade Católica do Rio de Janeiro, Departamento de Matemática.

Inclui bibliografia

1. Matemática - Dissertação. 2. Equação de Isaacs;. 3. Teoria de regularidade;. 4. Operadores de Bellman;. 5. Regularidade em espaços de Sobolev;. 6. Regularidade em espaços de Hölder; . I. Pimentel, Edgard. II. Pontifícia Universidade Católica do Rio de Janeiro. Departamento de Matemática. III. Título. 


\section{Acknowledgments}

I would like to first thank my advisor, for his guidance, support, motivation and trust in me throughout this work.

I also like to thank all members of my examination committee, for accepting the invitations and for the corrections and comments made.

I am very grateful to all professors for the lessons at PUC, and all administrative staff for the help and the kindness given to me.

My sincere thanks to my family and Costa Rican friends for the constant support since I begin this course.

Then I wish to thank the Graduate Program in Mathematics at PUC-Rio and CNPq-Brazil for the support. 


\section{Abstract}

Walker Ureña, Miguel Beltran; Pimentel, Edgard (Advisor). Regularity transmission by approximation methods: the Isaacs equation. Rio de Janeiro, 2019. 58p. Dissertação de mestrado - Departamento de Matemática, Pontifícia Universidade Católica do Rio de Janeiro.

Isaacs equation is an important example of fully nonlinear elliptic equation, appearing in a wide of disciplines. Of particular interest is the fact that such equations are driven by nonconvex operators. Therefore, it falls off the scope of the Evans-Krylov theory and poses additional, delicate, challenges when it comes to its regularity theory. We describe a series of recent results on the regularity theory of the Isaacs equation. These cover estimates in Hölder and Sobolev spaces. We argue through a genuinely geometrical method, by importing information from a related Bellman equation.

\section{Keywords}

Isaacs equation; Regularity theory; Bellman operators; Regularity in Sobolev spaces; Regularity in Hölder spaces; 


\section{Resumo}

Walker Ureña, Miguel Beltran; Pimentel, Edgard. Teoria de regularidade por métodos de aproximação: a equação de Isaacs. Rio de Janeiro, 2019. 58p. Dissertação de Mestrado - Departamento de Matemática, Pontifícia Universidade Católica do Rio de Janeiro.

A equação de Isaacs é um exemplo importante de equação elíptica totalmente não-linear, aparecendo em uma grande variedade de disciplinas. Um fato de interesse particular é que tais equações são dirigidas por operadores não convexos. Portanto, são compatíveis com a teoria de EvansKrylov e apresentam delicados desafios quando se trata de sua teoria da regularidade. Descrevemos uma série de resultados recentes sobre a teoria da regularidade da Equação de Isaacs. Estas cobrem estimativas nos espaços Hölder e Sobolev. Argumentamos através de um método genuinamente geométrico, importando informações de uma equação de Bellman relacionada.

\section{Palavras-chave}

Equação de Isaacs; Teoria de regularidade; Operadores de Bellman; Regularidade em espaços de Sobolev; Regularidade em espaços de Hölder; 


\section{Table of contents}

1 Background $\quad 11$

1.1 The Krylov-Safonov and Evans-Krylov theories 12

$\begin{array}{lll}1.2 & \text { Approximation Technique } & 16\end{array}$

1.3 Pucci Extremal Operators 20

1.4 Preliminaries on Sobolev Regularity 22

2 Estimates in Sobolev Spaces $\quad 31$

2.1 First Approximation Lemma 31

2.1.1 Sobolev Regularity 39

2.2 Second Approximation Lemma 41

2.2.1 Sobolev Regularity 42

3 Estimates in Hölder Spaces $\quad 44$

3.1 Approximation Lemma 44

3.2 Estimates in Log-Lipschitz Space 45

3.3 Estimates in Hölder Space $\quad 52$

$\begin{array}{ll}\text { Bibliography } & 58\end{array}$ 


\section{List of Abbreviations}

In what follows we put forward a list of notations used throughout this text. This is mostly based on [(E10), Appendix A.3].

\section{Basic Notation:}

(i) The $d$-dimensional Euclidean space is

$$
\mathbb{R}^{d}=\left\{\left(x_{1}, x_{2}, \ldots, x_{n}\right) \mid x_{i} \in \mathbb{R}, \forall i=1,2, \ldots, d\right\}
$$

with norms

$$
\left\|\left(x_{1}, x_{2}, \ldots, x_{n}\right)\right\|=\sqrt{x_{1}^{2}+x_{2}^{2}+\cdots+x_{n}^{2}}
$$

and

$$
\left\|\left(x_{1}, x_{2}, \ldots, x_{n}\right)\right\|_{\infty}=\max \left\{\left|x_{1}\right|,\left|x_{2}\right|, \ldots,\left|x_{n}\right|\right\}
$$

(ii) Open ball of radius $r$ :

$$
B_{r}\left(x_{0}\right)=\left\{x \in \mathbb{R}^{n} \mid\left\|x-x_{0}\right\|<r\right\} \quad \text { and } \quad B_{r}=B_{r}(0) .
$$

(iii) Open cube of side-length $r$ :

$$
Q_{r}\left(x_{0}\right)=\left\{x \in \mathbb{R}^{n} \mid\left\|x-x_{0}\right\|_{\infty}<\frac{r}{2}\right\} \quad \text { and } \quad Q_{r}=Q_{r}(0) \text {. }
$$

(iv) Lebesgue measure of a set $\mathcal{O}$ is denoted $|\mathcal{O}|$ or $\mathcal{L}(\mathcal{O})$.

Function Spaces and their norms: Let $\mathcal{O} \subseteq \mathbb{R}^{d}$.

(i) $\mathcal{C}(\mathcal{O})=\{u: \mathcal{O} \rightarrow \mathbb{R} \mid u$ continuous $\}$.

$\mathcal{C}(\overline{\mathcal{O}})=\{u: \mathcal{O} \rightarrow \mathbb{R} \mid u$ uniformly continuous $\}$.

If $\mathcal{O}$ is a bounded set, $C(\overline{\mathcal{O}})$ is a Banach space with

$$
\|u\|_{\mathcal{C}(\overline{\mathcal{O}})}=\sup _{x \in \mathcal{O}}|u(x)|
$$


(ii) $\mathcal{C}^{k}(\mathcal{O})=\{u: \mathcal{O} \rightarrow \mathbb{R} \mid u$ is $k$-times continuously differentiable $\}$.

$\mathcal{C}^{k}(\overline{\mathcal{O}})=\left\{u \in \mathcal{C}^{k}(\mathcal{O}) \mid D^{\alpha} u\right.$ is uniformly continuous for all $\left.|\boldsymbol{\alpha}| \leq k\right\}$

where $\boldsymbol{\alpha}=\left(\alpha_{1}, \alpha_{2}, \ldots, \alpha_{n}\right) \in \mathbb{N}^{n}$ and

$$
D^{\alpha} u=\frac{\partial^{|\alpha|} u}{\partial x_{1}^{\alpha_{1}} \cdots \partial x_{1}^{\alpha_{n}}},|\boldsymbol{\alpha}|=\alpha_{1}+\alpha_{2}+\cdots+\alpha_{n}
$$

If $\mathcal{O}$ is a bounded set, $C^{k}(\overline{\mathcal{O}})$ is a Banach space with

$$
\|u\|_{\mathcal{C}^{k}(\overline{\mathcal{O}})}=\sup _{|\boldsymbol{\alpha}| \leq k} \sup _{x \in \mathcal{O}}\left|D^{\alpha} u(x)\right|
$$

We also denote,

$$
\mathcal{C}^{\infty}(\mathcal{O})=\bigcap_{k=0}^{\infty} \mathcal{C}^{k}(\mathcal{O}) \text { and } \quad C^{\infty}(\overline{\mathcal{O}})=\bigcap_{k=0}^{\infty} C^{k}(\overline{\mathcal{O}})
$$

(iii) We denote $\mathcal{C}_{c}(\mathcal{O}), \mathcal{C}_{c}^{k}(\mathcal{O}), \ldots$, the spaces comprised of $\mathcal{C}(\mathcal{O}), \mathcal{C}^{k}(\mathcal{O}), \ldots$ functions with compact support.

(iv) If $0<\gamma \leq 1, \mathcal{C}^{0, \gamma}(\overline{\mathcal{O}})$ is the space of Hölder continuous functions with exponent $\gamma$, or $\gamma^{\text {th }}$-Hölder continuous. That is,

$$
\mathcal{C}^{0, \gamma}(\overline{\mathcal{O}})=\left\{u \in C(\overline{\mathcal{O}})|\exists C<\infty,| f(x)-f(y)|\leq C| x-\left.y\right|^{\gamma}, \forall x, y \in \mathcal{O}\right\}
$$

$\mathcal{C}^{0,1}$ is also called Lipschitz space, and if $\gamma \neq 1$ we can write $\mathcal{C}^{\gamma}=C^{0, \gamma}$.

In this case we have the seminorm

$$
[u]_{\gamma, \mathcal{O}}=[u]_{C^{0, \gamma}(\overline{\mathcal{O}})}=\sup _{\substack{x, y \in \overline{\mathcal{O}} \\ x \neq y}}\left\{\frac{|u(x)-u(y)|}{|x-y|^{\gamma}}\right\},
$$

and the $\gamma^{\text {th }}$-Hölder norm

$$
\|u\|_{C^{0, \gamma}(\overline{\mathcal{O}})}=\|u\|_{C(\overline{\mathcal{O}})}+[u]_{\gamma, \mathcal{O}}
$$

In general, $\mathcal{C}^{k, \gamma}(\overline{\mathcal{O}})$ is the $\gamma^{\text {th }}$-Hölder space

$$
\mathcal{C}^{k, \gamma}(\overline{\mathcal{O}})=\left\{u \in \mathcal{C}(\overline{\mathcal{O}}) \mid\|u\|_{C^{k, \gamma}(\overline{\mathcal{O}})}<\infty\right\}
$$

where

$$
\|u\|_{C^{k, \gamma}(\overline{\mathcal{O}})}=\sum_{|\alpha| \leq k}\left\|D^{\alpha} u\right\|_{C(\overline{\mathcal{O}})}+\sum_{|\alpha|=k}\left[D^{\alpha} u\right]_{\gamma, \mathcal{O}} .
$$


The $\gamma^{\text {th }}$-Hölder Spaces are also Banach Spaces.

(v) $L^{p}(\mathcal{O})=\left\{u: \mathcal{O} \rightarrow \mathbb{R} \mid u\right.$ is Lebesgue measurable and $\left.\|u\|_{L^{p}(\mathcal{O})}<\infty\right\}$, where for $1 \leq p<\infty$

$$
\|u\|_{L^{p}(\mathcal{O})}=\left(\int_{\mathcal{O}}|u(x)|^{p} d x\right)^{\frac{1}{p}}
$$

$L^{\infty}(\mathcal{O})=\left\{u: \mathcal{O} \rightarrow \mathbb{R} \mid u\right.$ is Lebesgue measurable and $\left.\|u\|_{L^{\infty}(\mathcal{O})}<\infty\right\}$, where

$$
\|u\|_{L^{\infty}(\mathcal{O})}=\underset{x \in \mathcal{O}}{\operatorname{ess} \sup }|u(x)|=\inf \{C \in \mathbb{R}|| f(x) \mid \leq C \text { a.e. on } \mathcal{O}\}
$$

(vi) $p$ - $\operatorname{BMO}(\mathcal{O})$ is the $p^{t h}$-bounded mean oscillation space of functions $f \in$ $L_{\text {loc }}^{1}(\mathcal{O})$, with norm

$$
\|f\|_{\mathrm{BMO}(\mathcal{O})}=\sup _{B_{r}(x) \subset \mathcal{O}}\left\{\left(f_{B_{r}(x)}\left|f(y)-\langle f\rangle_{x, r}\right|^{p} d y\right)^{1 / p}\right\}<\infty,
$$

where $\langle f\rangle_{x, r}$ is the average value of $f$ in $B_{r}(x)$ :

$$
\langle f\rangle_{x, r}=\langle f\rangle_{B_{r}(x)}=f_{B_{r}(x)} f(y) d y=\frac{1}{\left|B_{r}(x)\right|} \int_{B_{r}(x)} f(y) d y .
$$

We also denote $\langle f\rangle=\langle f\rangle_{0,1}$ and $m(f)(x)=\sup _{r>0}\langle f\rangle_{x, r}$.

(vii) $W^{k, p}(\mathcal{O})=\left\{u \in L^{p}(\mathcal{O}) \mid D^{\alpha} u \in L^{p}(\mathcal{O}), \forall \boldsymbol{\alpha} \in \mathbb{N}^{n}\right.$ s.t. $\left.|\boldsymbol{\alpha}| \leq k\right\}$ is the Sobolev space.

We have the norm

$$
\|u\|_{W^{k, p}}(\mathcal{O})=\left[\sum_{0 \leq|\boldsymbol{\alpha}| \leq k}\left\|D^{\alpha} u\right\|_{L^{p}(\mathcal{O})}^{p}\right]^{1 / p} \quad \text { in the case } 1 \leq p<\infty,
$$

and we have

$$
\|u\|_{W^{k, \infty}(\mathcal{O})}=\max _{0 \leq|\alpha| \leq k}\left\|D^{\alpha} u\right\|_{L^{\infty}(\mathcal{O})}
$$

We also denote $H^{k}=W^{k, 2}$.

(viii) $W_{0}^{k, p}(\mathcal{O})$ is the closure of $C_{c}^{\infty}(\mathcal{O})$ in $W^{k, p}(\mathcal{O})$, so

$$
W_{0}^{k, p}(\mathcal{O})=\left\{u \in W^{k, p}(\mathcal{O}) \mid \exists\left(u_{n}\right)_{n \in \mathbb{N}} \text { s.t. } u_{n} \in C_{c}^{\infty}(\mathcal{O}) \text { and }\left\|u_{n}-u\right\|_{W^{k, p}(\mathcal{O})} \rightarrow 0\right\} .
$$




\section{Background}

The Isaacs equation appeared in the context of two-players zero-sum (stochastic) differential games (Ruffus Isaacs, circa 1960), where an important reference is (I65).

The goal of this work is to examine the regularity of the solutions to the Isaacs equation. We argue by importing information from a related problem, namely the Bellman equation. Both are particular examples, introduced in Remark 1.2 (page 14), of uniform elliptic fully nonlinear problems.

The strategy used is how follow: given a problem of interest we look for an auxiliary model, from which information can be imported; examples of this are,

- $\Delta u$ for $\operatorname{Tr}\left(A(x) D^{2} u\right)$, in the basic PDE theory;

- $F\left(D^{2} u, x\right)$ for $F\left(D^{2} u, x_{0}\right)$, in Fully non linear PDE theory.

We argue through approximation techniques; the principal ingredients of the strategy are, first, a preliminary compactness level that usually is the $\mathcal{C}^{0, \alpha_{-}}$ estimates; Next an approximation lemma, which import information from the Bellman equation; Finally an iteration argument inspired on Caffarelli (Ann. Math., 89).

Here, we examine results reported in (P19), and mostly of the theoretical foundations are inspired by the so-called Caffarelli's regularity theory. We study regularity for weak (viscosity) solutions of fully nonlinear second order elliptic equations. For that reason, we introduce here some relevant definitions and results. See also (C89) and (CC95).

The second chapter studies the Sobolev regularity for the Isaacs equation, following the tools introduced at the end the first one, where Lemma 2.1 (first approximation lemma) is the principal tool, and Theorem $\mathbf{2 . 5}\left(W^{2, p}\right.$ regularity) is the first principal result; next Theorem 2.8 establishes a generalization.

The last chapter focuses on the Hölder regularity. Here, the main results are the $\mathcal{C}_{\text {loc }}^{1, \text { Log-Lip }}$ regularity (Theorem 3.4) and the $\mathcal{C}^{2, \gamma}$ regularity (Theorem $3.7)$. 


\section{1}

\section{The Krylov-Safonov and Evans-Krylov theories}

In this section we collect basic facts and definitions. In particular, we present the Krylov-Safonov and the Evans-Krylov theories. We starts with a definition.

Definition 1.1 (Uniform Elliptic Operator) If $\mathcal{S}(d)$ is the set of real symmetric matrices of dimension $d \times d$ and $0<\lambda \leq \Lambda$, an operator $F: \mathcal{S}(d) \rightarrow \mathbb{R}$ is called $(\lambda, \Lambda)$-elliptic if for all $M, N \in \mathcal{S}(d)$ such that $N \geq 0$, we have

$$
\lambda\|N\| \leq F(M+N)-F(M) \leq \Lambda\|N\|,
$$

where $\|N\|$ is the spectral norm (maximum eigenvalue of $N$ ).

Definition 1.2 Consider a fully nonlinear second order elliptic equation of the form

$$
F\left(D^{2} u, x\right)=f(x) \quad \text { in } \quad B_{1} \subset \mathbb{R}^{d}
$$

where $F: \mathcal{S}(d) \times B_{1} \rightarrow \mathbb{R}$ is a $(\lambda, \Lambda)$-elliptic operator and $f: B_{1} \rightarrow \mathbb{R}$ is continuous.

(i) A function $u \in \mathcal{C}\left(B_{1}\right)$ is a viscosity subsolution to (1-1) if for all $\varphi \in \mathcal{C}^{2}\left(B_{1}\right)$ such that $u-\varphi$ has a local maximum at $x_{0} \in B_{1}$, we have

$$
F\left(D^{2} \varphi\left(x_{0}\right), x_{0}\right) \geq f\left(x_{0}\right)
$$

We also say that

$$
F\left(D^{2} u, x\right) \geq f(x)
$$

in the viscosity sense.

(ii) A function $u \in \mathcal{C}\left(B_{1}\right)$ is a viscosity supersolution to (1-1) if for all $\varphi \in \mathcal{C}^{2}\left(B_{1}\right)$ such that $u-\varphi$ has a local minimum at $x_{0} \in B_{1}$, we have

$$
F\left(D^{2} \varphi\left(x_{0}\right), x_{0}\right) \leq f\left(x_{0}\right)
$$

We also say that

$$
F\left(D^{2} u, x\right) \leq f(x)
$$

in the viscosity sense.

(iii) A function $u \in \mathcal{C}\left(B_{1}\right)$ is a viscosity solution to (1-1) if it is both a viscosity subsolution and supersolution of (1-1). 
Next we recall an result on the stability of the viscosity solutions.

Theorem 1.1 Let $\left\{F_{k}\right\}_{k \geq 0}$ be a sequence of $(\lambda, \Lambda)$-elliptic operators and $\left\{u_{k}\right\}_{k \geq 0} \subseteq \mathcal{C}\left(B_{1}\right)$ be such that

$$
F_{k}\left(D^{2} u_{k}, x\right)=f(x) \quad \text { in } \quad B_{1}
$$

in the viscosity sense. If $\left\{F_{k}\right\}$ converges uniformly in compact sets and $\left\{u_{k}\right\}$ is uniformly bounded in compact sets of $B_{1}$, then there exist $u \in C\left(B_{1}\right)$ and a subsequence of $\left\{u_{k}\right\}$ that converges uniformly to $u$ in compact sets of $B_{1}$ and

$$
F\left(D^{2} u, x\right)=f(x) \quad \text { in } \quad B_{1},
$$

in the viscosity sense.

Proof. This is [(CC95), Proposition 4.11], proved by an application of the Arzelà-Ascoli Theorem.

Definition 1.3 (Uniform Elliptic Matrices) A symmetric matrix $A$ : $B_{1} \rightarrow \mathbb{R}^{d^{2}}$ is called $(\lambda, \Lambda)$-elliptic if

$$
\lambda I \leq A(x) \leq \Lambda I
$$

for every $x \in B_{1}$, or what is the same

$$
\lambda\|\xi\|^{2} \leq \sum_{i, j} a_{i, j}(x) \xi_{i} \xi_{j} \leq \Lambda\|\xi\|^{2} \quad, \forall \xi \in \mathbb{R}^{d}
$$

uniformly on $x \in B_{1}$, being $A=\left[a_{i, j}\right]_{i, j=1,2, \ldots d}$.

It follows that the operators

$$
F(M):=\operatorname{Tr}\left(\bar{A}_{\beta}(x) M\right)
$$

and

$$
G(M, x):=\sup _{\alpha \in \mathcal{A}} \inf _{\beta \in \mathcal{B}}\left[-\operatorname{Tr}\left(A_{\alpha, \beta}(x) M\right)\right]
$$

are $(\lambda, \Lambda)$-elliptic, when $\bar{A}_{\beta}$ and $A_{\alpha, \beta}$ are uniform $(\lambda, \Lambda)$-elliptic.

Remark 1.1 We say that $F(M, x)$ is convex in $M \in \mathcal{S}(d)$ if

$$
F\left(t M_{1}+(1-t) M_{2}\right) \leq t F\left(M_{1}, x\right)+(1-t) F\left(M_{2}, x\right)
$$


for every $t \in[0,1]$ and $M_{1}, M_{2} \in \mathcal{S}(d)$. We also have that, $F(M, x)$ is concave in $M$ if

$$
F\left(t M_{1}+(1-t) M_{2}\right) \geq t F\left(M_{1}, x\right)+(1-t) F\left(M_{2}, x\right)
$$

We also note that, if $F(M, x)$ is convex in $M$, then $-F(M, x)$ is concave with respect to $M$.

Remark 1.2 Some particular examples on which we have interest are:

(a) The Bellman Equation

$$
\inf _{\beta \in \mathcal{B}}\left[-\operatorname{Tr}\left(\bar{A}_{\beta}(x) D^{2} u\right)\right]=f(x) \quad \text { in } \quad B_{1}
$$

where $\bar{A}_{\beta}: B_{1} \times \mathcal{B} \rightarrow \mathbb{R}^{d^{2}}$ is a $(\lambda, \Lambda)$-elliptic matrix. In this case, it corresponds to the form (1-1) when

$$
F(M, x):=\inf _{\beta \in \mathcal{B}}\left[-\operatorname{Tr}\left(\bar{A}_{\beta}(x) M\right)\right]
$$

Thus, for all $M_{1}, M_{2} \in \mathcal{S}(d)$

$$
\begin{aligned}
F( & \left.t M_{1}+(1-t) M_{2}, x\right) \\
& =\inf _{\beta \in \mathcal{B}}\left[-\operatorname{Tr}\left(\bar{A}_{\beta}(x)\left(t M_{1}+(1-t) M_{2}\right)\right)\right] \\
& =\inf _{\beta \in \mathcal{B}}\left[-t \operatorname{Tr}\left(\bar{A}_{\beta}(x) M_{1}\right)-(1-t) \operatorname{Tr}\left(\bar{A}_{\beta}(x) M_{2}\right)\right] \\
& \leq t \inf _{\beta \in \mathcal{B}}\left[-\operatorname{Tr}\left(\bar{A}_{\beta}(x) M_{1}\right)\right]+(1-t) \inf _{\beta \in \mathcal{B}}\left[-\operatorname{Tr}\left(\bar{A}_{\beta}(x) M_{2}\right)\right],
\end{aligned}
$$

then we have

$$
F\left(t M_{1}+(1-t) M_{2}\right) \leq t F\left(M_{1}, x\right)+(1-t) F\left(M_{2}, x\right)
$$

implies that $F(M, x)$ is a $(\lambda, \Lambda)$-elliptic convex operator in $M$. Therefore, many regularity results as the Evans-Krylov Theory can be applied to it.

(b) A general form of a Bellman Equation is

$$
\inf _{\beta \in \mathcal{B}}\left[-\operatorname{Tr}\left(\bar{A}_{\beta}(x) D^{2} u\right)-\mathbf{b}_{\beta}(x) \cdot D u-f_{\beta}(x)\right]=0 \quad \text { in } \quad B_{1},
$$

where $\bar{A}_{\beta}: B_{1} \times \mathcal{B} \rightarrow \mathbb{R}^{d^{2}}$ is a $(\lambda, \Lambda)$-elliptic matrix, the coefficient $\mathbf{b}_{\beta}: B_{1} \times \mathcal{B} \rightarrow \mathbb{R}^{d}$ is a vector field such that $\mathbf{b}_{\beta} \in L^{p}\left(B_{1}\right)$ uniformly and $f_{\beta}: B_{1} \times \mathcal{B} \rightarrow \mathbb{R}$ is a real function. 
(c) The Isaacs Equation

$$
\sup _{\alpha \in \mathcal{A}} \inf _{\beta \in \mathcal{B}}\left[-\operatorname{Tr}\left(A_{\alpha, \beta}(x) D^{2} u\right)\right]=f(x) \quad \text { in } \quad B_{1}
$$

where $A_{\alpha, \beta}: B_{1} \times \mathcal{A} \times \mathcal{B} \rightarrow \mathbb{R}^{d^{2}}$ is a $(\lambda, \Lambda)$-elliptic matrix. In this case, it corresponds to the form (1-1) when

$$
F(M, x):=\sup _{\alpha \in \mathcal{A}} \inf _{\beta \in \mathcal{B}}\left[-\operatorname{Tr}\left(A_{\alpha, \beta}(x) M\right)\right]
$$

that in general, is neither convex nor concave with respect to $M$.

(d) A general form of an Issacs Equation is

$$
\sup _{\alpha \in \mathcal{A}} \inf _{\beta \in \mathcal{B}}\left[-\operatorname{Tr}\left(A_{\alpha, \beta}(x) D^{2} u\right)-\mathbf{b}_{\alpha, \beta}(x) \cdot D u-f_{\alpha, \beta}(x)\right]=0 \quad \text { in } \quad B_{1},
$$

where $A_{\alpha, \beta}: B_{1} \times \mathcal{A} \times \mathcal{B} \rightarrow \mathbb{R}^{d^{2}}$ is a $(\lambda, \Lambda)$-elliptic matrix, the coefficient $\mathbf{b}_{\alpha, \beta}: B_{1} \times \mathcal{A} \times \mathcal{B} \rightarrow \mathbb{R}^{d}$ is a vector field such that $\mathbf{b}_{\alpha, \beta} \in L^{p}\left(B_{1}\right)$ uniformly and $f_{\alpha, \beta}: B_{1} \times \mathcal{A} \times \mathcal{B} \rightarrow \mathbb{R}$ is a real function.

We close this section which two foundational results.

Theorem 1.2 (Krylov-Safonov Theorem) Let $F: S(d) \rightarrow \mathbb{R}$ be $a(\lambda, \Lambda)$ elliptic operator and $u \in \mathcal{C}\left(B_{1}\right)$ a viscosity solution to $F\left(D^{2} u\right)=0$ in $B_{1}$. Then $u \in \mathcal{C}_{\text {loc }}^{1, \alpha}\left(B_{1}\right)$ and

$$
\|u\|_{\mathcal{C}^{1, \alpha}\left(\bar{B}_{1 / 2}\right)} \leq C\left(\|u\|_{L^{\infty}\left(B_{1}\right)}+|F(0)|\right)
$$

for some $\alpha \in(0,1)$ and $C>0$, universal constants.

Proof. It is [(CC95), Corollary 5.7]

Theorem 1.3 (Evans-Krylov Theorem) Let $u \in \mathcal{C}\left(B_{1}\right)$ be a viscosity solution to $F\left(D^{2} u\right)=0$ in $B_{1}$, where $F: S(d) \rightarrow \mathbb{R}$ is a concave or convex $(\lambda, \Lambda)$-elliptic operator. Then $u \in \mathcal{C}_{\text {loc }}^{2, \alpha}\left(B_{1}\right)$ and

$$
\|u\|_{\mathcal{C}^{2, \alpha}\left(\bar{B}_{1 / 2}\right)} \leq C\left(\|u\|_{L^{\infty}\left(B_{1}\right)}+|F(0)|\right)
$$

for some $\alpha \in(0,1)$ and $C>0$ universal constants.

Proof. See [(CC95), Theorem 6.6] 


\section{2}

\section{Approximation Technique}

In order to illustrate the approximation technique used in this work, we will present its application proving the Hölder regularity of the gradient of the solutions to $F\left(D^{2} u\right)=f$ in $B_{1}$, with $f \in L^{p}\left(B_{1}\right)$.

We start with an approximation lemma.

Lemma 1.4 (An approximation Lemma) Let $u \in \mathcal{C}\left(B_{1}\right)$ be a viscosity solution of $F\left(D^{2} u\right)=f$ in $B_{1}$, where $F: S(d) \rightarrow \mathbb{R}$ is a $(\lambda, \Lambda)$-elliptic operator and $f \in L_{\mathrm{loc}}^{p}\left(B_{1}\right)$, with $p>d$. For any $\delta>0$ there exist $\epsilon>0$ and $h \in \mathcal{C}_{\mathrm{loc}}^{1, \alpha}\left(B_{1}\right)$, for some $\alpha \in(0,1)$, such that if $\|f\|_{L^{p}\left(B_{1}\right)}<\epsilon$, then

$$
\|u-h\|_{L^{\infty}\left(\bar{B}_{1}\right)}<\delta .
$$

Proof. Suppose by contradiction that the statement of this lemma is false. Then there are sequences $\left(F_{n}\right)_{n \in \mathbb{N}},\left(f_{n}\right)_{n \in \mathbb{N}}$ and $\left(u_{n}\right)_{n \in \mathbb{N}}$ such that $F_{n}$ is $(\lambda, \Lambda)$-elliptic, $f_{n} \rightarrow 0$ in $L^{p}\left(B_{1}\right)$ and $u_{n} \in \mathcal{C}\left(B_{1}\right)$, such that $u_{n}$ is a viscosity solution to

$$
F_{n}\left(D^{2} u_{n}\right)=f_{n} \quad \text { in } \quad B_{1}
$$

with

$$
\left\|u_{n}-h\right\|_{L^{\infty}\left(B_{1}\right)}>\delta_{0} \quad, \forall h \in C_{\mathrm{loc}}^{1, \alpha}\left(B_{1}\right),
$$

for some $\delta_{0}>0$. Since $F_{n}$ is uniformly elliptic, $F_{n}$ is also uniformly Lipchitz; then it converges to some operator $F_{\infty}$. By application of Theorem 1.1, there exist $u \in C\left(B_{1}\right)$ and a subsequence of $\left\{u_{n}\right\}$ that converges uniformly to $u$ in compact sets of $B_{1}$. Note that $F_{\infty}$ is still $(\lambda, \Lambda)$-elliptic, $u_{\infty} \in \mathcal{C}\left(B_{1}\right)$ and

$$
F_{\infty}\left(D^{2} u_{\infty}\right)=0 \quad \text { in } \quad B_{1}
$$

so by the Theorem 1.2, $u_{\infty} \in C^{1, \alpha}\left(B_{1}\right)$, for some $\alpha \in(0,1)$. Thus, if we take $h=u_{\infty}, h \in C^{1, \alpha}\left(B_{1}\right)$ and there exists $N>0$ such that

$$
\left\|u_{n}-h\right\|_{L^{\infty}\left(B_{1}\right)}=\left\|u_{n}-u_{\infty}\right\|_{L^{\infty}\left(B_{1}\right)}<\delta_{0},
$$

but this is a contradiction.

Remark 1.3 Given $\gamma \in(0,1)$ and a bounded open set $\mathcal{O} \subset \mathbb{R}^{d}$, we say that $u \in \mathcal{C}^{k, \gamma}(\overline{\mathcal{O}})$ when there exists a polynomial $P$ of degree $k$ such that

$$
|u(x)-P(x)| \leq C\left\|x-x_{0}\right\|^{k+\gamma} \quad, \forall x, x_{0} \in \mathcal{O} .
$$


Particularly, $u \in \mathcal{C}_{\text {loc }}^{k, \gamma}\left(B_{1}\right)$ if, for some $\rho \in(0,1)$, there exists $C>0$ such that, for every $n \in \mathbb{N}$ and any $x_{0} \in B_{1}$

$$
\sup _{\left\|x-x_{0}\right\|<\rho^{n}}\left|u(x)-P_{n}(x)\right| \leq C \rho^{n(k+\gamma)}
$$

for some polynomials $P_{n}$ of degree $k$.

Lemma 1.5 Let $F: S(d) \rightarrow \mathbb{R}$ be a $(\lambda, \Lambda)$-elliptic operator and $f \in L_{\mathrm{loc}}^{\infty}\left(B_{1}\right)$. If $u$ is a viscosity solution of the equation

$$
F\left(D^{2} u\right)=f \quad \text { in } \quad B_{1}
$$

then there exist $\bar{\alpha} \in(0,1)$ and $h \in \mathcal{C}_{\text {loc }}^{1, \bar{\alpha}}\left(B_{1}\right)$ such that

$$
\sup _{x \in B_{\rho}}|u(x)-h(0)-D h(0) \cdot x| \leq \rho^{1+\alpha} \quad, \forall \alpha \in(0, \bar{\alpha})
$$

for some $\rho \in(0,1)$.

Proof. By the Lemma 1.4, for any $\delta>0$ and some $\bar{\alpha} \in(0,1)$, there exists $h \in \mathcal{C}_{\text {loc }}^{1, \bar{\alpha}}\left(B_{1}\right)$ such that

$$
\|u-h\|_{L^{\infty}\left(B_{1}\right)}<\delta
$$

and by properties of the Taylor polynomial, for some $\rho \in(0,1)$,

$$
\sup _{x \in B_{\rho}}|h(x)-h(0)-D h(0) \cdot x|<C \rho^{1+\bar{\alpha}}
$$

Then, for all $x \in B_{\rho}$

$$
\begin{aligned}
\mid u(x)-h(0)-D h(0) \cdot x) \mid & \leq|u(x)-h(x)|+|h(x)-h(0)-D h(0) \cdot x| \\
& \leq \delta+C \rho^{1+\bar{\alpha}} .
\end{aligned}
$$

Fix $\alpha \in(0, \bar{\alpha})$ and take

$$
\delta=\frac{\rho^{1+\alpha}}{2} \text { and } \rho=\left(\frac{1}{2 C}\right)^{\frac{1}{\bar{\alpha}-\alpha}}
$$

Thus we obtain

$$
\sup _{x \in B_{\rho}}|u(x)-h(0)-D h(0) \cdot x| \leq \rho^{1+\alpha} \quad, \forall \alpha \in(0, \bar{\alpha}) .
$$


Lemma 1.6 Let $F: S(d) \rightarrow \mathbb{R}$ be a $(\lambda, \Lambda)$-elliptic operator and $f \in L_{\mathrm{loc}}^{p}\left(B_{1}\right)$. If $u$ is a viscosity solution of the equation $F\left(D^{2} u\right)=f$ in $B_{1}$ and $\|u\|_{\mathcal{C}\left(\bar{B}_{1}\right)} \leq 1$, then there are $C>0, \bar{\alpha} \in(0,1), a_{n} \in \mathbb{R}$ and $\mathbf{b}_{n} \in \mathbb{R}^{d}$ satisfying

$$
\sup _{x \in B_{\rho^{n}}}\left|u(x)-a_{n}-\mathbf{b}_{n} \cdot x\right| \leq \rho^{n(1+\alpha)}
$$

and

$$
\left|a_{n}-a_{n-1}\right|+\rho^{n-1}|| \mathbf{b}_{n}-\mathbf{b}_{n-1} \| \leq C \rho^{(n-1)(1+\alpha)},
$$

for any $\rho \in(0,1)$ and $n \in \mathbb{N}$.

Proof. We proceed by induction in $n \in \mathbb{N}$. In the basic case, take $a_{0}=a_{-1}=0$ and $\mathbf{b}_{0}=\mathbf{b}_{-1}=0$, which satisfy the conditions for $n=0$.

Now, we suppose the case $n$ is satisfied, and set $v_{n}: B_{1} \rightarrow \mathbb{R}$ as

$$
v_{n}(x):=\frac{u\left(\rho^{n} x\right)-a_{n}-\mathbf{b}_{n} \cdot\left(\rho^{n} x\right)}{\rho^{n(1+\alpha)}}
$$

Then

$$
D^{2} v_{n}(x)=\frac{\rho^{2 n} D^{2} u\left(\rho^{n} x\right)}{\rho^{n(1+\alpha)}}=\rho^{n(1-\alpha)} D^{2} u\left(\rho^{n} x\right),
$$

which implies

$$
F\left(\frac{D^{2} v_{n}(x)}{\rho^{n(1-\alpha)}}\right)=F\left(D^{2} u\left(\rho^{n} x\right)\right)=f\left(\rho^{n} x\right)
$$

If we define

$$
F_{n}(M):=\rho^{n(1-\alpha)} F\left(\frac{M}{\rho^{n(1-\alpha)}}\right) \quad \text { and } \quad f_{n}(x):=\rho^{n(1-\alpha)} f\left(\rho^{n} x\right),
$$

then we get that $v_{n}$ solves $F_{n}\left(D^{2} v_{n}\right)=f_{n}(x)$ in $B_{1}$. So, by the Lemma 1.5 there exist $h \in \mathcal{C}^{1, \bar{\alpha}}\left(B_{1}\right)$ and $C>0$ such that

$$
\begin{aligned}
\rho^{1+\alpha} & >\sup _{x \in B_{\rho}}\left|v_{n}(x)-h(0)-D h(0) \cdot x\right| \\
& =\sup _{x \in B_{\rho}}\left|\frac{u\left(\rho^{n} x\right)-a_{n}-\mathbf{b}_{n} \cdot\left(\rho^{n} x\right)}{\rho^{n(1+\alpha)}}-h(0)-D h(0) \cdot x\right| \\
& =\sup _{x \in B_{\rho^{n+1}}}\left|\frac{u(x)-a_{n}-\mathbf{b}_{n} \cdot x}{\rho^{n(1+\alpha)}}-h(0)-D h(0) \cdot\left(\rho^{-n} x\right)\right| .
\end{aligned}
$$

Then we obtain,

$$
\begin{aligned}
\sup _{x \in B_{\rho^{n+1}}} & \left|u(x)-a_{n}-\mathbf{b}_{n} \cdot x-\rho^{n(1+\alpha)} h(0)-\rho^{n(1+\alpha)} D h(0) \cdot\left(\rho^{-n} x\right)\right| \\
& <\rho^{1+\alpha} \cdot \rho^{n(1+\alpha)} .
\end{aligned}
$$


Thus, by setting

$$
a_{n+1}:=a_{n}+\rho^{n(1+\alpha)} h(0) \quad \text { and } \quad \mathbf{b}_{n+1}:=\mathbf{b}_{n}+\rho^{n \alpha} D h(0),
$$

we have that

$$
\sup _{x \in B_{\rho^{n}}}\left|u(x)-a_{n+1}-\mathbf{b}_{n+1} \cdot x\right|<\rho^{(n+1)(1+\alpha)} .
$$

Moreover, there exists $C>0$ such that

$$
\begin{aligned}
\left|a_{n+1}-a_{n}\right|+\rho^{n}\left\|\mathbf{b}_{n+1}-\mathbf{b}_{n}\right\| & =\rho^{n(1+\alpha)}|h(0)|+\rho^{n(1+\alpha)}\|D h(0)\| \\
& \leq C \rho^{n(1+\alpha)}
\end{aligned}
$$

Hence, the statement is true in the case $n+1$, completing the induction argument.

To illustrate the strategy behind regularity transmission by approximation methods, we establish the Krylov-Safonov theory in the non-homogeneous setting.

Theorem 1.7 Let $F: S(d) \rightarrow \mathbb{R}$ be a $(\lambda, \Lambda)$-elliptic operator and $f \in$ $L_{\mathrm{loc}}^{p}\left(B_{1}\right)$. If $u$ is a viscosity solution of the equation $F\left(D^{2} u\right)=f$ in $B_{1}$ and $\|u\|_{\mathcal{C}\left(\bar{B}_{1}\right)} \leq 1$, then there is $\bar{\alpha} \in(0,1)$ such that $u \in \mathcal{C}_{\text {loc }}^{1, \alpha}\left(B_{1}\right)$, for any $\alpha \in(0, \bar{\alpha})$.

Proof. By the Lemma 1.6, we have that there are $C>0, \bar{\alpha} \in(0,1), a_{n} \in \mathbb{R}$ and $\mathbf{b}_{n} \in \mathbb{R}^{d}$ satisfying

$$
\sup _{x \in B_{\rho^{n}}}\left|u(x)-a_{n}-\mathbf{b}_{n} \cdot x\right| \leq \rho^{n(1+\alpha)}
$$

and

$$
\left|a_{n}-a_{n-1}\right|+\rho^{n-1} \| \mathbf{b}_{n}-\mathbf{b}_{n-1}|| \leq C \rho^{(n-1)(1+\alpha)},
$$

for any $\rho \in(0,1)$ and $n \in \mathbb{N}$. Then we have

$$
\left|a_{n}-u(0)\right|=\left|u(0)-P_{n}(0)\right| \leq \rho^{n(1+\alpha)},
$$

and also

$$
\begin{aligned}
\left|\mathbf{b}_{m}-\mathbf{b}_{n}\right| & \leq C \sum_{k=n+1}^{m}\left|\mathbf{b}_{k}-\mathbf{b}_{k-1}\right| \\
& \leq C \sum_{k=n+1}^{m} \rho^{(k-1) \alpha} \\
& =C \rho^{n \alpha} \cdot \frac{1-\rho^{(m-n) \alpha}}{1-\rho^{\alpha}} \\
& \leq \widetilde{C} \rho^{n} .
\end{aligned}
$$


for any $m>n$. Then $a_{n}$ and $\mathbf{b}_{n}$ are convergent because $\rho \in(0,1)$, thus they are bounded. Then $a_{n}$ and $\mathbf{b}_{n}$ are bounded because At the end we have that $u$ is $C_{\text {loc }}^{1, \alpha}\left(B_{1}\right)$ at 0 . Next, by changes of variables we can conclude $u \in C_{\text {loc }}^{1, \alpha}\left(B_{1}\right)$.

\section{3}

\section{Pucci Extremal Operators}

The Pucci extremal operators described in this section are important for the theory of fully nonlinear elliptic equations. They allow us to define the class of uniform viscosity solutions in order to simplify some proofs.

Definition 1.4 (Pucci extremal operators) The Pucci extremal operators $\mathcal{M}_{\lambda, \Lambda}^{ \pm}: \mathcal{S}(d) \rightarrow \mathbb{R}$ are defined as follow:

$$
\mathcal{M}_{\lambda, \Lambda}^{-}(M):=\inf _{A \in S_{\lambda, \Lambda}(d)} \operatorname{Tr}(A M)=\Lambda \sum_{e_{i}<0} e_{i}+\lambda \sum_{e_{i}>0} e_{i}
$$

and

$$
\mathcal{M}_{\lambda, \Lambda}^{+}(M):=\sup _{A \in S_{\lambda, \Lambda}(d)} \operatorname{Tr}(A M)=\Lambda \sum_{e_{i}>0} e_{i}+\lambda \sum_{e_{i}<0} e_{i}
$$

where $e_{i}$ are the eigenvalues of $M$ and $S_{\lambda, \Lambda}(d)$ is the set of symmetric matrices whose eigenvalues belong to $[\lambda, \Lambda]$.

Remark 1.4 Note that $\mathcal{M}_{\lambda, \Lambda}^{+}$and $\mathcal{M}_{\lambda, \Lambda}^{-}$are concave and convex operators respectively. The equations

$$
\mathcal{M}_{\lambda, \Lambda}^{+}\left(D^{2} u\right)=f(x) \quad \text { and } \quad \mathcal{M}_{\lambda, \Lambda}^{-}\left(D^{2} u\right)=f(x)
$$

are called Pucci equations and they are examples of nonlinear uniformly elliptic equations.

Definition 1.5 (Viscosity solutions) Let $f \in \mathcal{C}\left(B_{1}\right)$. We say that $u \in$ $\mathcal{C}\left(B_{1}\right)$ is in the class of supersolutions $\bar{S}(\lambda, \Lambda, f)$ if

$$
\mathcal{M}_{\lambda, \Lambda}^{-}\left(D^{2} u\right) \leq f \quad \text { in } \quad B_{1}
$$

in the viscosity sense.

Similarly, $u \in \mathcal{C}\left(B_{1}\right)$ is in the class of subsolutions $\underline{S}(\lambda, \Lambda, f)$ if

$$
\mathcal{M}_{\lambda, \Lambda}^{+}\left(D^{2} u\right) \geq f \quad \text { in } \quad B_{1}
$$

in the viscosity sense. 
Finally the class of $(\lambda, \Lambda)$-viscosity solutions is the set

$$
S(\lambda, \Lambda, f)=\bar{S}(\lambda, \Lambda, f) \cap \underline{S}(\lambda, \Lambda, f) .
$$

Remark 1.5 Noting that

$$
\mathcal{M}_{\lambda, \Lambda}^{+}(M)=-M_{\lambda, \Lambda}^{-}(-M)
$$

and, for any $(\lambda, \Lambda)$-elliptic operator $F$

$$
\mathcal{M}_{\lambda, \Lambda}^{-}\left(D^{2} u\right) \leq F\left(D^{2} u\right) \leq \mathcal{M}_{\lambda, \Lambda}^{+}\left(D^{2} u\right)
$$

we have that $\pm u \in S(\lambda, \Lambda, \pm f)$, when $F\left(D^{2} u\right)=f$. for any $(\lambda, \Lambda)$-uniformly elliptic operator $F$.

Lemma 1.8 We have:

(i) $\lambda^{\prime} \leq \lambda \leq \Lambda \leq \Lambda^{\prime} \Longrightarrow \underline{S}(\lambda, \Lambda, f) \subset \underline{S}\left(\lambda^{\prime}, \Lambda^{\prime}, f\right)$; the same holds for $\bar{S}$ and $S$.

(ii) $u \in \underline{S}(\lambda, \Lambda, f) \Longrightarrow u \in \bar{S}(\lambda, \Lambda,-f)$.

(iii) $u \in \underline{S}(\lambda, \Lambda, f)$, then for all $\alpha, r>0$

$$
v(y):=\alpha u\left(\frac{y}{r}\right) \Longrightarrow v \in S\left(\lambda, \Lambda, \frac{\alpha}{r^{2}} f\left(\frac{\cdot}{r}\right)\right) .
$$

Theorem 1.9 Let $F: S(d) \times B_{1} \rightarrow \mathbb{R}$ be a $(\lambda, \Lambda)$-elliptic operator and $u \in \mathcal{C}\left(B_{1}\right)$. If $F\left(D^{2} u, x\right) \geq f(x)\left[\right.$ resp. $\left.F\left(D^{2} u, x\right) \leq f(x)\right]$ in the viscosity sense in $B_{1}$, then

$$
u-\phi \in \underline{S}\left(\frac{\lambda}{d}, \Lambda, f(x)-F\left(D^{2} \phi(x), x\right)\right)
$$

for any $\phi \in \mathcal{C}^{2}\left(B_{1}\right)$.

$$
\left[\text { resp. } u-\phi \in \bar{S}\left(\frac{\lambda}{d}, \Lambda, f(x)-F\left(D^{2} \phi(x), x\right)\right)\right]
$$

Proof. Follows from [(CC95), Proposition 2.13]

Theorem 1.10 (Interior Hölder regularity) If $u \in S(\lambda, \Lambda, f)$ in $B_{1}$, then $u \in \mathcal{C}^{\alpha}\left(\bar{B}_{1 / 2}\right)$ and

$$
\|u\|_{\mathcal{C}^{\alpha}\left(B_{1 / 2}\right)} \leq C\left(\|u\|_{L^{\infty}\left(B_{1}\right)}+\|f\|_{L^{d}\left(B_{1}\right)}\right)
$$

for some $\alpha \in(0,1)$ and $C>0$ universal constants. 
Proof. Follows from [(CC95), Proposition 4.10 and Remark 5]

\section{4}

\section{Preliminaries on Sobolev Regularity}

Here we present the basic elements in the study of Sobolev regularity.

Definition 1.6 Let $\mathcal{O} \subset \mathbb{R}^{d}$ be a $\mathcal{C}^{1,1}$ bounded domain, i.e., for each point $x \in \partial \mathcal{O}$ there is a neighborhood $\mathcal{O}_{x}$ such that $\partial\left(\mathcal{O} \cap \mathcal{O}_{x}\right)$ is the graph of a function of class $\mathcal{C}^{1,1}\left(\overline{\mathcal{O}_{x}}\right)$.

(i) A convex paraboloid of opening $M \in[0,+\infty)$ is a function $P_{M}^{+}: \mathcal{O} \rightarrow \mathbb{R}$ of the form

$$
P_{M}^{+}(x)=\ell(x)+\frac{M}{2}\|x\|^{2},
$$

where $\ell: \mathcal{O} \rightarrow \mathbb{R}$ is an affine function.

In the same way, a concave paraboloid of opening $M \in[0,+\infty)$ is a function $P_{M}^{-}: \mathcal{O} \rightarrow \mathbb{R}$ of the form

$$
P_{M}^{-}(x)=\ell(x)-\frac{M}{2}\|x\|^{2} .
$$

(ii) We define

$\bar{\Theta}(u, \mathcal{O})(x):=\inf \left\{M \in \mathbb{R} \mid \exists P_{M}^{+}, P_{M}^{+}(x)=u(x) \wedge P_{M}^{+}(y) \geq u(y), \forall y \in \mathcal{O}\right\}$

the infimum of all openings of the convex paraboloids touching $u$ from above at $x$ in $\mathcal{O}$, and

$\underline{\Theta}(u, \mathcal{O})(x):=\inf \left\{M \in \mathbb{R} \mid \exists P_{M}^{-}, P_{M}^{-}(x)=u(x) \wedge P_{M}^{-}(y) \leq u(y), \forall y \in \mathcal{O}\right\}$,

the infimum of all openings of the concave paraboloids touching $u$ from below at $x$ in $\mathcal{O}$. We also have

$$
\Theta(u, \mathcal{O})(x):=\max \{\bar{\Theta}(u, \mathcal{O})(x), \underline{\Theta}(u, \mathcal{O})(x)\}
$$

The existence of $P_{M}^{ \pm}$touching the viscosity solution is ensured by [(CC95), Proposition 2.4], which states by maximum principle, that viscosity solutions in certain bounded domains, containing $x$ are touched by paraboloids from below and above, in an open neighborhood of $x$.

Lemma 1.11 Let $1<p \leq \infty, 0<r<\infty, u \in \mathcal{C}\left(B_{r}\right)$ and $\Theta\left(u, B_{r}\right) \in L^{p}\left(B_{r}\right)$. Then $D^{2} u \in L^{p}\left(B_{r}\right)$. 
Proof. We follow [(CC95),Proposition 1.1]. The proof starts from the fact that

$$
-\underline{\Theta}\left(u, B_{\delta}\right)(x) \leq \Delta_{\delta \mathbf{e}}^{2} u(x) \leq \bar{\Theta}\left(u, B_{\delta}\right)(x),
$$

where

$$
\Delta_{h}^{2} u(x):=\frac{u(x+h)+u(x-h)-2 u(x)}{\|h\|^{2}} .
$$

For any $\varphi \in \mathcal{C}^{\infty}\left(B_{r}\right)$,

$$
\int_{\mathbb{R}^{d}} u \frac{\partial^{2} \varphi}{\partial \mathbf{e}^{2}} d x=\lim _{\delta \rightarrow 0} \int_{\mathbb{R}^{d}} u \Delta_{\delta \mathbf{e}}^{2} \varphi d x=\lim _{\delta \rightarrow 0} \int_{\mathbb{R}^{d}} \varphi \Delta_{\delta \mathbf{e}}^{2} u d x
$$

Since $\left|\Delta_{\delta \mathbf{e}}^{2} u\right| \leq \Theta\left(u, B_{r}\right) \in L^{p}\left(B_{r}\right)$,

$$
\left|\int_{B_{r}} \varphi \Delta_{\delta \mathbf{e}}^{2} u d x\right| \leq\|\varphi\|_{L^{p^{\prime}\left(B_{r}\right)}}\left\|\Theta\left(u, B_{r}\right)\right\|_{L^{p}\left(B_{r}\right)}<\infty .
$$

Therefore, by the Dominated Convergence Theorem, there exists

$$
v=\lim _{\delta \rightarrow 0} \Delta_{\delta \mathbf{e}}^{2} u \in L^{p}\left(B_{r}\right)
$$

such that

$$
\int_{\mathbb{R}^{d}} u \frac{\partial^{2} \varphi}{\partial \mathbf{e}^{2}} d x=\int_{\mathbb{R}^{d}} \phi v d x
$$

Now, noting that

$$
\varphi_{i j}=\partial_{\frac{e_{i}+e_{j}}{2}}, \frac{e_{i}+e_{j}}{2} \varphi-\frac{\varphi_{i i}}{2}-\frac{\varphi_{j j}}{2},
$$

we have

$$
\left|\int_{B_{r}} u \varphi_{i j} d x\right| \leq 2\|\varphi\|_{L^{p^{\prime}\left(B_{r}\right)}}\left\|\Theta\left(u, B_{r}\right)\right\|_{L^{p}\left(B_{r}\right)}<\infty
$$

which implies

$$
u \in W^{2, p}\left(B_{r}\right) \Longleftrightarrow D^{2} u \in L^{p}\left(B_{r}\right)
$$

Lemma 1.12 Let $p \in\left(\frac{d}{2},+\infty\right)$ and $\mathcal{O}$ be a $\mathcal{C}^{1,1}$ bounded domain. If $u \in$ $W^{2, p}(\mathcal{O})$, we have

$$
\|\Theta(u, \mathcal{O})\|_{L^{p}(\mathcal{O})} \leq C\|u\|_{W^{2, p}(\mathcal{O})}
$$

where $C=C(d, p)$ is a nonnegative constant.

Proof. This proof is based on (LZ15) and [(CCS96),Appendix C]). Let $\tilde{u}$ be the extension of $u$ to $\mathbb{R}^{n}$ and $x_{0} \in \mathcal{O}$ be a Lebesgue point of $\tilde{u}, D \tilde{u}$ and $D^{2} \tilde{u}$. We define $h: \mathbb{R}^{n} \rightarrow \mathbb{R}$ such that

$$
h(x)=\tilde{u}(x)-\left[\tilde{u}\left(x_{0}\right)+D \tilde{u}\left(x_{0}\right)\left(x-x_{0}\right)+\frac{1}{2}\left(x-x_{0}\right)^{T} D^{2} \tilde{u}\left(x_{0}\right)\left(x-x_{0}\right)\right] .
$$


If $q^{*}=\frac{d q}{q-d}>d$ for some $q \geq 1$ s.t. $q \leq p<d$, and $r=\left\|x-x_{0}\right\|$, we have

$$
\begin{aligned}
|h(x)| & =\frac{r}{r^{d / q^{*}}} \frac{\left|h(x)-h\left(x_{0}\right)\right|}{\left\|x-x_{0}\right\|^{1-d / q^{*}}} \\
& \leq \frac{r\left|B_{1}\right|^{1 / q^{*}}}{\left|B_{r}\left(x_{0}\right)\right|^{1 / q^{*}}}[h]_{C^{0,1-d / q^{*}}}
\end{aligned}
$$

Next, as $[h]_{C^{0,1-d / q^{*}}} \leq\|h\|_{C^{0,1-d / q^{*}}}$, by Morrey's inequality there exists a universal constant $C_{1}>0$ such that

$$
\begin{aligned}
|h(x)| & \leq \frac{r}{\left|B_{r}\left(x_{0}\right)\right|^{1 / q^{*}}} \cdot C_{1}\|D h\|_{L^{q^{*}}\left(B_{r}\left(x_{0}\right)\right)} \\
& =\frac{C_{1} r}{\left|B_{r}\left(x_{0}\right)\right|^{1 / q^{*}}}\left(\int_{B_{r}\left(x_{0}\right)}\|D h(y)\|^{q^{*}} d y\right)^{1 / q^{*}} \\
& \leq C_{1} r\left[\left(f_{B_{r}\left(x_{0}\right)}\left\|D h-\langle D h\rangle_{B r\left(x_{0}\right)}\right\|^{q^{*}}\right)^{1 / q^{*}}+\left(f_{B_{r}\left(x_{0}\right)}\left\|\langle D h\rangle_{B r\left(x_{0}\right)}\right\|^{q *}\right)^{1 / q^{*}}\right],
\end{aligned}
$$

because of the Minkowski inequality. Now we get

$$
|h(x)| \leq C_{1} r\left(\int_{B_{r}\left(x_{0}\right)}\left\|D h-\langle D h\rangle_{B_{r}\left(x_{0}\right)}\right\|^{q^{*}}\right)^{1 / q^{*}}+C_{1} r\langle D h\rangle_{B_{r}\left(x_{0}\right)} .
$$

After that, by Poincaré's inequality there is a constant $C_{2}>0$ such that

$$
\left(f_{B_{r}(x)}\left\|D h(y)-\langle D h\rangle_{B_{r}(x)}\right\|^{q^{*}} d y\right)^{1 / q^{*}} \leq C_{2} r\left(f_{B_{r}(x)}\left\|D^{2} h\right\|^{q}\right)^{1 / q} .
$$

As $D^{2} h(x)=D^{2} \tilde{u}(x)-D^{2} \tilde{u}\left(x_{0}\right)$, then if $C=\max \left\{C_{1}, C_{1} C_{2}\right\}$, we obtain

$$
\begin{aligned}
|h(x)| \leq C r^{2}\left(f_{B_{r}\left(x_{0}\right)}\left\|D^{2} \tilde{u}(y)-D^{2} \tilde{u}\left(x_{0}\right)\right\|^{q} d y\right)^{1 / q}+C_{1} r\langle D h\rangle_{B_{r}\left(x_{0}\right)} \\
\leq C r^{2}\left[\left(f_{B_{r}\left(x_{0}\right)}\left\|D^{2} \tilde{u}(y)\right\|^{q} d y\right)^{1 / q}+\left(f_{B_{r}\left(x_{0}\right)}\left\|D^{2} \tilde{u}\left(x_{0}\right)\right\|^{q} d y\right)^{1 / q}\right] \\
\quad+C_{1} r\langle D h\rangle_{B_{r}\left(x_{0}\right)} \\
=C r^{2}\left[\left(f_{B_{r}\left(x_{0}\right)}\left\|D^{2} \tilde{u}(y)\right\|^{q} d y\right)^{1 / q}+\left\|D^{2} \tilde{u}\left(x_{0}\right)\right\|\right]+\langle D h\rangle_{B_{r}\left(x_{0}\right)} \\
\leq C r^{2}\left[m\left(\left\|D^{2} \tilde{u}\right\|^{q}\right)^{1 / q}\left(x_{0}\right)+\left\|D^{2} \tilde{u}\left(x_{0}\right)\right\|\right]+\langle D h\rangle_{B_{r}\left(x_{0}\right) .}
\end{aligned}
$$


Note that, there is $C>0$ (replace the actual $C$, if it is greater) such that

$$
\begin{aligned}
\langle D h\rangle_{B_{r}\left(x_{0}\right)} & =f_{B_{r}\left(x_{0}\right)} D h(y) d y \\
& \leq C f_{B_{r}\left(x_{0}\right)} D^{2} h(y) d y \\
& =C f_{B_{r}\left(x_{0}\right)}\left\|D^{2} \tilde{u}(y)-D^{2} \tilde{u}\left(x_{0}\right)\right\| d y \\
& \leq C \sup _{0<s \leq r} f_{B_{s}\left(x_{0}\right)}\left\|D^{2} \tilde{u}(y)\right\| d y+C\left\|D^{2} \tilde{u}\left(x_{0}\right)\right\| \\
& \leq C m\left(\left\|D^{2} \tilde{u}\right\|\right)\left(x_{0}\right)+C\left\|D^{2} \tilde{u}\left(x_{0}\right)\right\|,
\end{aligned}
$$

therefore we obtain, for almost every $x_{0} \in \mathcal{O}$ (and replacing $C+1$ for $C$ ), that

$$
|h(x)| \leq C r^{2}\left[m\left(\left\|D^{2} u\right\|^{q}\right)^{1 / q}\left(x_{0}\right)+m\left(\left\|D^{2} u\right\|\right)\left(x_{0}\right)+\left\|D^{2} u\left(x_{0}\right)\right\|\right] .
$$

Since $D^{2} u \in L^{p}(\mathcal{O})$

$$
\left\|m\left(\left\|D^{2} u\right\|\right)\right\|_{L^{p}(\mathcal{O})} \leq C\left\|D^{2} u\right\|_{L^{p}(\mathcal{O})}<\infty
$$

We also have that $p \geq q$ and $\left\|D^{2} u\right\|^{q} \in L^{p / q}(\mathcal{O})$, then

$$
\begin{aligned}
\left\|m\left(\left\|D^{2} u\right\|^{q}\right)^{1 / q}\right\|_{L^{p}(\mathcal{O})} & =\left\|m\left(\left\|D^{2} u\right\|^{q}\right)\right\|_{L^{p / q}(\mathcal{O})} \\
& \leq C\|\| D^{2} u\left\|^{q}\right\|_{L^{p / q}(\mathcal{O})} \\
& =C\left\|D^{2} u\right\|_{L^{p}(\mathcal{O})}
\end{aligned}
$$

Thus

$$
m\left(\left\|D^{2} u\right\|^{q}\right)^{1 / q}\left(x_{0}\right)+m\left(\left\|D^{2} u\right\|\right)\left(x_{0}\right)+\left\|D^{2} u\left(x_{0}\right)\right\| \in L^{p}(\mathcal{O}) .
$$

At the end, we have for a.e $x_{0} \in \mathcal{O}$, since $r=\left\|x-x_{0}\right\|$,

$$
\begin{aligned}
\left|u(x)-u\left(x_{0}\right)\right| \leq & \left\|D u\left(x_{0}\right)\right\| \cdot r+\frac{1}{2}\left\|D^{2} u\left(x_{0}\right)\right\| \cdot r^{2} \\
& +C\left[m\left(\left\|D^{2} u\right\|^{q}\right)^{\frac{1}{q}}\left(x_{0}\right)+m\left(\left\|D^{2} u\right\|\right)\left(x_{0}\right)+\left\|D^{2} u\left(x_{0}\right)\right\|\right] \cdot r^{2} .
\end{aligned}
$$

Then, according to the definition of $\Theta$, for a.e $x_{0} \in \mathcal{O}$

$$
\begin{aligned}
\Theta(u, \mathcal{O})\left(x_{0}\right) \leq \| & D^{2} u\left(x_{0}\right) \| \\
& +2 C\left[m\left(\left\|D^{2} u\right\|^{q}\right)^{1 / q}\left(x_{0}\right)+m\left(\left\|D^{2} u\right\|\right)\left(x_{0}\right)+\left\|D^{2} u\left(x_{0}\right)\right\|\right] .
\end{aligned}
$$

This implies $\Theta(u, \mathcal{O}) \in L^{p}(\mathcal{O})$. 
Definition 1.7 Using the notation of Definition 1.6 , we set

$$
G_{M}(u, \mathcal{O}):=\bar{G}_{M}(u, \mathcal{O}) \cap \underline{G}_{M}(u, \mathcal{O}),
$$

where

$$
\bar{G}_{M}(u, \mathcal{O}):=\left\{x \in \mathcal{O} \mid \exists P_{M}^{+}, P_{M}^{+}(x)=u(x) \text { and } P_{M}^{+}(y) \geq u(y), \forall y \in \mathcal{O}\right\}
$$

and

$$
\underline{G}_{M}(u, \mathcal{O}):=\left\{x \in \mathcal{O} \mid \exists P_{M}^{-}, P_{M}^{-}(x)=u(x) \text { and } P_{M}^{-}(y) \leq u(y), \forall y \in \mathcal{O}\right\} .
$$

We also define,

$$
\begin{aligned}
& \bar{A}_{M}(u, \mathcal{O})=\mathcal{O} \backslash \bar{G}_{M}(u, \mathcal{O}), \\
& \underline{A}_{M}(u, \mathcal{O})=\mathcal{O} \backslash \underline{G}_{M}(u, \mathcal{O})
\end{aligned}
$$

and

$$
A_{M}(u, \mathcal{O})=\mathcal{O} \backslash G_{M}(u, \mathcal{O})
$$

Remark 1.6 According to the Definition 1.6 and the Definition 1.7, note that

$$
\bar{\Theta}(u, \mathcal{O})(x)=\inf \left\{M \in \mathbb{R} \mid x \in \bar{G}_{M}(u, \mathcal{O})\right\}
$$

and

$$
\underline{\Theta}(u, \mathcal{O})(x)=\inf \left\{M \in \mathbb{R} \mid x \in \underline{G}_{M}(u, \mathcal{O})\right\} .
$$

Lemma 1.13 If $\mathcal{O} \subseteq \mathbb{R}^{d}$ is a $\mathcal{C}^{1,1}$ bounded domain and $u \in W^{2, p}(\mathcal{O})$, there exists $C>0$ such that

$$
\left|A_{t}(u, \mathcal{O})\right| \leq C t^{-p}
$$

Proof. Note that

$$
A_{t}(u, \mathcal{O})=\{\Theta(u, \mathcal{O})>t\}=\{x \in \mathcal{O} \mid \Theta(u, \mathcal{O})(x)>t\}
$$

That is because

$$
\begin{aligned}
x \in A_{t}(u, \mathcal{O}) & \Longleftrightarrow x \notin G_{t}(u, \mathcal{O})=\bar{G}_{t}(u, \mathcal{O}) \cap \underline{G}_{t}(u, \mathcal{O}) \\
& \Longleftrightarrow x \notin \bar{G}_{t}(u, \mathcal{O}) \quad \text { or } \quad x \notin \underline{G_{t}}(u, \mathcal{O}) \\
& \Longleftrightarrow \bar{\Theta}(u, \mathcal{O})(x)>t \quad \text { or } \quad \underline{\Theta}(u, \mathcal{O})(x)>t \\
& \Longleftrightarrow \Theta(u, \mathcal{O})(x)=\max \{\bar{\Theta}(u, \mathcal{O})(x), \underline{\Theta}(u, \mathcal{O})(x)\}>t \\
& \Longleftrightarrow x_{0} \in\{\Theta(u, \mathcal{O})>t\} .
\end{aligned}
$$


So by Markov's inequality

$$
\begin{aligned}
\left|A_{t}(u, \mathcal{O})\right|=|\{\Theta(u, \mathcal{O})>t\}| & \leq \frac{1}{t^{p}} \int_{\mathcal{O}}|\Theta(u, \mathcal{O})(x)|^{p} d x \\
& \leq t^{-p}\|\Theta(u, \mathcal{O})\|_{L^{p}(\mathcal{O})}^{p}
\end{aligned}
$$

By Lemma 1.12, $\Theta(u, \mathcal{O}) \in L^{p}(\mathcal{O})$. Then, there exists $C>0$ such that

$$
\left|A_{t}(u, \mathcal{O})\right| \leq C t^{-p}
$$

Lemma 1.14 (Calderón-Zygmund cube decomposition) Let $A \subset B \subset$ $Q_{1}$ be measurable sets and $\sigma \in(0,1)$. For a dyadic cube $K \subset Q_{1}$, denote by $\bar{K}$ its predecessor. If $|A| \leq \sigma$ and

$$
|A \cap K| \geq \sigma|K| \Longrightarrow \bar{K} \subset B
$$

then we obtain

$$
|A| \leq \sigma|B|
$$

Proof. By the Calderón-Zygmund technique, we can choose a sequence of dyadic cubes $K^{1}, K^{2}, \ldots$, such that

$$
A \subset \bigcup_{i=1}^{\infty} K^{i} \text { a.e. } \quad, \quad \frac{\left|K^{i} \cap A\right|}{\left|K^{i}\right|}>\sigma \quad \text { and } \quad \frac{\left|\bar{K}^{i} \cap A\right|}{\left|K^{i}\right|} \leq \sigma
$$

and such that $\left(\overline{K^{i}}\right)_{i=1,2, \ldots}$ are pairwise disjoint. We have that

$$
\left|A \cap K^{i}\right| \geq \sigma\left|K^{i}\right| \Longrightarrow \overline{K^{i}} \subset B \Longrightarrow \bigcup_{i=1}^{\infty} \overline{K^{i}} \subset B
$$

then

$$
\begin{aligned}
|A| & \leq \sum_{i=1}^{\infty}\left|K^{i} \cap A\right| \\
& \leq \sum_{i=1}^{\infty}\left|\overline{K^{i}} \cap A\right| \\
& \leq \sigma \sum_{i=1}^{\infty}\left|\overline{K^{i}}\right| \\
& =\sigma\left|\bigcup_{i=1}^{\infty} \overline{K^{i}}\right| \\
& \leq \sigma|B| .
\end{aligned}
$$


Lemma 1.15 Let $g: \mathcal{O} \rightarrow[0, \infty)$ be a measurable function. For $t>0$, define

$$
\mu_{g}(t):=|\{x \in \mathcal{O} \mid g(x)>t\}|
$$

Fix $\nu>0$ and $M>1$; for $p>1$, define

$$
\mathcal{S}:=\sum_{k=1}^{\infty} M^{p k} \mu_{g}\left(\nu M^{k}\right)
$$

Then

$$
g \in L^{p}(\mathcal{O}) \Longleftrightarrow \mathcal{S}<\infty
$$

Moreover, there exists a positive constant $C$ such that

$$
C^{-1} \mathcal{S} \leq\|g\|_{L^{p}(\mathcal{O})} \leq C(|\mathcal{O}|+\mathcal{S})
$$

Proof. Note that $\mu_{g}:[0, \infty) \rightarrow[0, \infty)$ is a decreasing function, so we can take a negative Lebesgue-Stieltjes measure

$$
\begin{aligned}
\mu([a, b)) & :=\mu_{g}(b)-\mu_{g}(a) \\
& =-|\{x \in \mathcal{O} \mid a \leq g(x) \leq b\}| \\
& =-\mathcal{L}\left(g^{-1}([a, b))\right) .
\end{aligned}
$$

Because the extension measure is unique, we have

$$
\mu(E)=\mathcal{L}\left(g^{-1}(E)\right)
$$

for all $E \subset \mathcal{O}$ measurable and $d \mu(t)=\mu_{g}^{\prime}(t) d t$. So, for all measurable $\phi:[0, \infty) \rightarrow[0, \infty)$

$$
\begin{aligned}
\int_{\mathcal{O}} \phi \circ g(x) d \mathcal{L}(x) & =\int_{0}^{\infty} \phi \circ g \circ g^{-1}(t) d \mathcal{L}\left(g^{-1}(t)\right) \\
& =-\int_{0}^{\infty} \phi(t) \mu_{g}^{\prime}(t) d t \\
& =-\left.\phi(t) \mu_{g}(t)\right|_{0} ^{\infty}+\int_{0}^{\infty} \phi^{\prime}(t) \mu_{g}(t) d t .
\end{aligned}
$$

In particular, if $\phi(t)=t^{p}$, we obtain

$$
\|g\|_{L^{p}(\mathcal{O})}=\int_{\mathcal{O}}|g(x)|^{p} d \mathcal{L}(x)=-\lim _{t \rightarrow \infty} t^{p} \mu_{g}(t)+\int_{0}^{\infty} p t^{p-1} \mu_{g}(t) d t .
$$

Since

$$
-\|g\|_{L^{p}(\mathcal{O})} \leq-t^{p} \mu_{g}(t) \leq 0
$$


and $M>1$ we have $M^{k}<M^{k+1} \rightarrow \infty$; then

$$
\begin{aligned}
2\|g\|_{L^{p}(\mathcal{O})}^{p} & \geq \int_{0}^{\nu M} p t^{p-1} \mu_{g}(t) d t+\sum_{k=1}^{\infty} \int_{\nu M^{k}}^{\nu M^{k+1}} p t^{p-1} \mu_{g}(t) d t . \\
& \geq \mu_{g}(\nu M) \int_{0}^{\nu M} p t^{p-1} d t+\sum_{k=1}^{\infty} \mu_{g}\left(\nu M^{k+1}\right) \int_{\nu M^{k}}^{\nu M^{k+1}} p t^{p-1} d t \\
& =\left.\mu_{h}(\nu M) t^{p}\right|_{0} ^{\nu M}+\left.\sum_{k=1}^{\infty} \mu_{g}\left(\nu M^{k+1}\right) t^{p}\right|_{\nu M^{k}} ^{\nu+1} \\
& =\mu_{h}(\nu M) \nu^{p} M^{p}+\sum_{k=1}^{\infty} \mu_{g}\left(\nu M^{k+1}\right) \nu^{p} M^{p k}\left(M^{p}-1\right) \\
& =\nu^{p} M^{p} \mu_{h}(\nu M)+\sum_{k=2}^{\infty} \mu_{g}\left(\nu M^{k}\right) \nu^{p} M^{p(k-1)}\left(M^{p}-1\right) \\
& \geq \nu^{p} \frac{M^{p}-1}{M^{p}} \sum_{k=1}^{\infty} M^{p k} \mu_{g}\left(\nu M^{k}\right) .
\end{aligned}
$$

On the other hand,

$$
\begin{aligned}
\|g\|_{L^{p}(\mathcal{O})}^{p} & \leq \mu_{g}(0) \int_{0}^{\nu M} p t^{p-1} d t+\sum_{k=1}^{\infty} \mu_{g}\left(\nu M^{k}\right) \int_{\nu M^{k}}^{\nu M^{k+1}} p t^{p-1} d t \\
& =\mu_{h}(0) \nu^{p} M^{p}+\sum_{k=1}^{\infty} \mu_{g}\left(\nu M^{k}\right) \nu^{p} M^{p k}\left(M^{p}-1\right) \\
& =|\mathcal{O}| \nu^{p} M^{p}+\nu^{p}\left(M^{p}-1\right) \sum_{k=1}^{\infty} M^{p k} \mu_{h}\left(\nu M^{k+1}\right) .
\end{aligned}
$$

As a consequence,

$$
\frac{\nu^{p}}{2} \frac{M^{p}-1}{M^{p}} \mathcal{S} \leq\|g\|_{L^{p}(\mathcal{O})}^{p} \leq \nu^{p} M^{p}(|\mathcal{O}|+\mathcal{S})
$$

so we can take

$$
C=\left\{\begin{array}{rl}
\frac{1}{2 \nu^{p}} \frac{M^{p}}{M^{p}-1} & , \text { if } M^{p}-1 \leq 1 \wedge \nu^{p} \leq \frac{1}{\nu^{p}} \\
\frac{\nu^{p}}{2} \frac{M^{p}}{M^{p}-1} & , \text { if } M^{p}-1 \leq 1 \wedge \nu^{p} \geq \frac{1}{\nu^{p}} \\
2 \nu^{p} M^{p} & , \text { if } M^{p}-1 \geq 1 \wedge \nu^{p} \geq \frac{1}{\nu^{p}} \\
\frac{2 M^{p}}{\nu^{p}} & , \text { if } M^{p}-1 \geq 1 \wedge \nu^{p} \leq \frac{1}{\nu^{p}} .
\end{array} .\right.
$$

Thus, there exists $C$ depending on $\nu, M$ and $p$ such that

$$
C^{-1} \mathcal{S} \leq\|g\|_{L^{p}(\mathcal{O})} \leq C(|\mathcal{O}|+\mathcal{S})
$$

and $g \in L^{\infty}(\mathcal{O})$ if and only if $\mathcal{S}<\infty$. 
Lemma 1.16 If $u \in S(\lambda, \Lambda, f)$ in $B_{1}$ with $\|f\|_{L^{q}\left(B_{1}\right)} \leq 1$ for some $q>d$, then there exist universal constants $C>0$ and $\mu>0$ such that

$$
\left|A_{t}\left(u, B_{1}\right) \cap Q_{1}\right| \leq C t^{-\mu}
$$

for every $t>0$.

Proof. Follows from [(LZ15), Lemma 3.2]. See also [(HL11), Lemma 5.15].

An important result we detail in the next chapter concerns the transmission of Sobolev regularity from the Bellman equation to the Isaacs equation. For that reason, we finish this chapter by examining the regularity of a Bellman homogeneous problem in $W^{2, p}$.

Theorem 1.17 ( $W^{2, q}$-regularity for the approximate problem) Let $r \in(0,1)$ and $d<p<q$. Let $h \in \mathcal{C}\left(B_{r}\right)$ be a viscosity solution to

$$
\inf _{\beta \in \mathcal{B}}\left[-\operatorname{Tr}\left(\bar{A}_{\beta}(x) D^{2} h\right)\right]=0 \quad \text { in } \quad B_{r}\left(x_{0}\right)
$$

where $\bar{A}_{\beta}: B_{1} \times \mathcal{A} \times \mathcal{B} \rightarrow \mathbb{R}^{d^{2}}$ is a $(\lambda, \Lambda)$-elliptic matrix. Suppose further that

$$
\left(f_{B_{r}\left(x_{0}\right)}\left|\sup _{\beta \in \mathcal{B}}\left\|\bar{A}_{\beta}(x)-\bar{A}_{\beta}\left(x_{0}\right)\right\|\right|^{d}\right)^{1 / d} \ll 1 / 2,
$$

for every $x_{0} \in B_{1}$. Then $h \in W^{2, q}\left(B_{r}\right) \cap \mathcal{C}\left(\bar{B}_{r^{+}}\right)$and there exists a universal constant $C>0$ such that $\|h\|_{W^{2, q}\left(B_{r}\right)} \leq C$.

Proof. Follows from [(CC95), Theorem 7.1]

Remark 1.7 Throughout this text, a constant is called universal, if it depends only on $d, \lambda$ and Lambda. 


\section{Estimates in Sobolev Spaces}

In this chapter we examine the Sobolev regularity for the Isaacs equation. To resort to results previously put forward here - especially Theorem 1.17 we assume

$$
\left(f_{B_{r}\left(x_{0}\right)}\left|\sup _{\beta \in \mathcal{B}}\left\|\bar{A}_{\beta}(x)-\bar{A}_{\beta}\left(x_{0}\right)\right\|\right|^{d}\right)^{1 / d} \ll 1 / 2 .
$$

It is well-known that such a smallness regime is usual when importing regularity from an auxiliary problem with constant coefficients.

\section{1}

\section{First Approximation Lemma}

We continue with an approximation lemma, instrumental in the establishment of our $W^{2, p}$-estimates for the Isaacs equation.

Lemma 2.1 (First Approximation Lemma) Let $u \in \mathcal{C}\left(B_{1}\right)$ be a viscosity solution to

$$
\sup _{\alpha \in \mathcal{A}} \inf _{\beta \in \mathcal{B}}\left[-\operatorname{Tr}\left(A_{\alpha, \beta}(x) D^{2} u\right)\right]=f(x) \quad \text { in } \quad B_{1} \text {, }
$$

where $A_{\alpha, \beta}: B_{1} \times \mathcal{A} \times \mathcal{B} \rightarrow \mathbb{R}^{d^{2}}$ is a $(\lambda, \Lambda)$-elliptic matrix. Suppose that $\bar{A}_{\beta}: B_{1} \times \mathcal{B} \rightarrow \mathbb{R}^{d^{2}}$ satisfies

$$
\|f\|_{L^{p}\left(B_{1}\right)}+\left\|A_{\alpha, \beta}-\bar{A}_{\beta}\right\|_{L^{\infty}\left(B_{1}\right)}<\epsilon_{1}
$$

for some $\epsilon_{1}>0$ to be determined further. Then, there exists $h \in W^{2, q}\left(B_{7 / 8}\right) \cap \mathcal{C}\left(\bar{B}_{8 / 9}\right)$ with $\|h\|_{W^{2, q}\left(B_{7 / 8}\right)} \leq C$, and

$$
\|u-h\|_{L^{\infty}\left(B_{7 / 8}\right)}+\|\xi\|_{L^{p}\left(B_{7 / 8}\right)} \leq C_{1}\left(\epsilon_{1}^{\gamma}\|u\|_{L^{\infty}\left(B_{1}\right)}+\|f\|_{L^{p}\left(B_{1}\right)}\right)
$$

where $C_{1}$ and $\gamma$ are non-negative constants depending on $(d, \lambda, \Lambda, C, q, p)$, and

$$
\xi(x)=f(x)-\sup _{\alpha \in \mathcal{A}} \inf _{\beta \in \mathcal{B}}\left[-\operatorname{Tr}\left(A_{\alpha, \beta}(x) D^{2} h(x)\right)\right]
$$

Proof. Let $h \in \mathcal{C}\left(\bar{B}_{8 / 9}\right)$ be the solution of the Dirichlet problem 


$$
\begin{cases}\inf _{\beta \in \mathcal{B}}\left[-\operatorname{Tr}\left(\bar{A}_{\beta}(x) D^{2} h(x)\right)\right]=0 & \text { in } \quad B_{8 / 9} \\ h=u & \text { on } \quad \partial B_{8 / 9} .\end{cases}
$$

Then by Theorem 1.17, $h \in W^{2, q}\left(B_{7 / 8}\right) \cap \mathcal{C}\left(B_{8 / 9}\right)$ and there exists $C$ such that $\|h\|_{W^{2, q}\left(B_{7 / 8}\right)} \leq C$. By the interior Hölder regularity from Theorem 1.10 and [(CC95), Propositions 4.12 and 4.13], there exists $C_{\bar{\gamma}}$ such that

$$
\|u\|_{\mathcal{C}^{\bar{\gamma}\left(\bar{B}_{8 / 9}\right)}} \leq C_{\bar{\gamma}}\left(\|u\|_{L^{\infty}\left(B_{1}\right)}+\|f\|_{L^{p}\left(B_{1}\right)}\right)
$$

and

$$
\|h\|_{\mathcal{C}_{\bar{\gamma} / 2}\left(\bar{B}_{8 / 9}\right)} \leq C_{\bar{\gamma}}\left(\|u\|_{L^{\infty}\left(B_{1}\right)}+\|f\|_{L^{p}\left(B_{1}\right)}\right),
$$

for some $\bar{\gamma} \in(0,1)$.

Let $\delta \in(0,1 / 2), x_{0} \in B_{8 / 9-\delta}$ and $x_{1} \in \partial B_{\delta}\left(x_{0}\right)$. By applying $W^{2, q}$ interior estimates to $h-h\left(x_{1}\right)$ in $B_{\delta}\left(x_{0}\right)$ when $d<p<q$, we have

$$
\left\|D^{2} h\right\|_{L^{q}\left(B_{\delta / 2}\left(x_{0}\right)\right)} \leq\left\|h-h\left(x_{1}\right)\right\|_{W^{2, q}\left(B_{\delta / 2}\left(x_{0}\right)\right)} \leq C_{\delta}\left\|h-h\left(x_{1}\right)\right\|_{L^{\infty}\left(\partial B_{\delta}\left(x_{0}\right)\right)},
$$

where $C_{\delta}=C_{h} \delta^{\frac{d-2 q}{q}}>C_{h}$, for some universal constant $C_{h}$. Note that for all $x \in B_{\delta}\left(x_{0}\right)$

$$
\left|h(x)-h\left(x_{1}\right)\right|=\left|x-x_{1}\right|^{\bar{\gamma} / 2} \frac{\left|h(x)-h\left(x_{1}\right)\right|}{\left|x-x_{1}\right|^{\bar{\gamma} / 2}} \leq(2 \delta)^{\bar{\gamma} / 2}\|h\|_{\mathcal{C}^{\bar{\gamma} / 2}\left(B_{\delta}\left(x_{0}\right)\right)} .
$$

Since $B_{\delta}\left(x_{0}\right) \subset B_{8 / 9}$, by (2-4) we obtain

$$
\begin{aligned}
\left\|D^{2} h\right\|_{L^{q}\left(B_{\delta / 2}\left(x_{0}\right)\right)} & \leq C_{\delta}(2 \delta)^{\bar{\gamma} / 2}\|h\|_{\mathcal{C}^{\bar{\gamma} / 2}\left(B_{\delta}\left(x_{0}\right)\right)} \\
& \leq C_{\delta}(2 \delta)^{\bar{\gamma} / 2} C_{\bar{\gamma}}\left(\|u\|_{L^{\infty}\left(B_{1}\right)}+\|f\|_{L^{p}\left(B_{1}\right)}\right) .
\end{aligned}
$$

Then

$$
\left\|D^{2} h\right\|_{L^{q}\left(B_{8 / 9-\delta}\right)} \leq \frac{\widetilde{C} C_{\delta}(2 \delta)^{\bar{\gamma} / 2} C_{\bar{\gamma}}}{\delta^{d}}\left(\|u\|_{L^{\infty}\left(B_{1}\right)}+\|f\|_{L^{p}\left(B_{1}\right)}\right),
$$

because there is $\widetilde{C}>0$ such that

$$
\left\|D^{2} h\right\|_{L^{q}\left(B_{8 / 9-\delta}\right)} \leq \frac{\widetilde{C}}{\delta^{d}}\left\|D^{2} h\right\|_{L^{q}\left(B_{\delta / 2}\left(x_{0}\right)\right)} .
$$

Note that, for all $\alpha \in \mathcal{A}, \beta \in \mathcal{B}, x_{0} \in B_{8 / 9-\delta}$, we have

$$
\begin{aligned}
\left|\operatorname{Tr}\left(\left(A_{\alpha, \beta}-\bar{A}_{\beta}\right)\left(x_{0}\right) D^{2} h\left(x_{0}\right)\right)\right| & \leq C(d)\left\|\left(A_{\alpha, \beta}-\bar{A}_{\beta}\right) D^{2} h\left(x_{0}\right)\right\| \\
& \leq C(d)\left\|\left(A_{\alpha, \beta}-\bar{A}_{\beta}\right)\left(x_{0}\right)\right\| \cdot\left\|D^{2} h\left(x_{0}\right)\right\| \\
& \leq \epsilon_{1} C(d)\left\|D^{2} h\left(x_{0}\right)\right\| .
\end{aligned}
$$


Hence

$$
\begin{aligned}
-\epsilon_{1} C(d)\left\|D^{2} h\left(x_{0}\right)\right\|- & \operatorname{Tr}\left(\bar{A}_{\beta} D^{2} h\left(x_{0}\right)\right) \\
\leq & -\operatorname{Tr}\left(A_{\alpha, \beta}\left(x_{0}\right) D^{2} h\left(x_{0}\right)\right) \\
& \leq \epsilon_{1} C(d)\left\|D^{2} h\left(x_{0}\right)\right\|-\operatorname{Tr}\left(\bar{A}_{\beta} D^{2} h\left(x_{0}\right)\right),
\end{aligned}
$$

and, since

$$
\inf _{\beta \in \mathcal{B}}\left[-\operatorname{Tr}\left(\bar{A}_{\beta}\left(x_{0}\right) D^{2} h\left(x_{0}\right)\right)\right]=0
$$

we get

$$
\left|\sup _{\alpha \in \mathcal{A}} \inf _{\beta \in \mathcal{B}}\left[-\operatorname{Tr}\left(A_{\alpha, \beta}\left(x_{0}\right) D^{2} h\left(x_{0}\right)\right)\right]\right| \leq \epsilon_{1} C(d)\left\|D^{2} h\left(x_{0}\right)\right\| .
$$

Thus we produce

$$
\begin{aligned}
& \| \sup _{\alpha \in \mathcal{A}} \inf _{\beta \in \mathcal{B}} {\left[-\operatorname{Tr}\left(A_{\alpha, \beta} D^{2} h\right)\right] \|_{L^{p}\left(B_{8 / 9-\delta}\right)} } \\
& \leq \epsilon_{1} \cdot C(d)\left\|D^{2} h\right\|_{L^{q}\left(B_{8 / 9-\delta}\right)} \\
& \leq \epsilon_{1} \cdot C(d) \frac{\widetilde{C} C_{\delta}(2 \delta)^{\bar{\gamma} / 2} C_{\bar{\gamma}}}{\delta^{d}}\left(\|u\|_{L^{\infty}\left(B_{1}\right)}+\|f\|_{L^{p}\left(B_{1}\right)}\right) \\
&=\epsilon_{1} \cdot \bar{C} \delta^{\frac{d-2 q}{q}+\frac{\bar{\gamma}}{2}-d}\left(\|u\|_{L^{\infty}\left(B_{1}\right)}+\|f\|_{L^{p}\left(B_{1}\right)}\right),
\end{aligned}
$$

where

$$
\bar{C}=\widetilde{C} C(d) C_{h} 2^{\bar{\gamma} / 2} C_{\bar{\gamma}}
$$

A consequence is

$$
\|\xi\|_{L^{p}\left(B_{8 / 9-\delta}\right)} \leq\|f\|_{L^{p}\left(B_{8 / 9-\delta}\right)}+\epsilon_{1} \cdot \bar{C} \delta^{\frac{d-2 q}{q}+\frac{\bar{\gamma}}{2}-d}\left(\|u\|_{L^{\infty}\left(B_{1}\right)}+\|f\|_{L^{p}\left(B_{1}\right)}\right) .
$$

By (2-3) and (2-4), noticing that $u-h=0$ in $\partial B_{8 / 9}$, and taking $y \in \partial B_{8 / 9}$, we conclude

$$
\begin{aligned}
\frac{u(x)-h(x)}{|x-y|^{\bar{\gamma} / 2}} & \leq\|u-h\|_{\mathcal{C}^{\bar{\gamma} / 2}\left(\bar{B}_{8 / 9}\right)} \\
& \leq\|u\|_{\mathcal{C}_{\bar{\gamma} / 2}\left(\bar{B}_{8 / 9}\right)}+\|h\|_{\mathcal{C}^{\bar{\gamma} / 2}\left(\bar{B}_{8 / 9}\right)} \\
& \leq\|u\|_{\mathcal{C}^{\bar{\gamma}}\left(\bar{B}_{8 / 9}\right)}+\|h\|_{\mathcal{C}^{\bar{\gamma} / 2}\left(\bar{B}_{8 / 9}\right)} \\
& \leq 2 C_{\bar{\gamma}}\left(\|u\|_{L^{\infty}\left(B_{1}\right)}+\|f\|_{L^{p}\left(B_{1}\right)}\right) .
\end{aligned}
$$

Then

$$
\frac{1}{\delta \bar{\gamma} / 2} \cdot\|u-h\|_{L^{\infty}\left(\bar{B}_{8 / 9-\delta}\right)} \leq 2 C_{\bar{\gamma}}\left(\|u\|_{L^{\infty}\left(B_{1}\right)}+\|f\|_{L^{p}\left(B_{1}\right)}\right) .
$$


If $\widehat{C}=\max \left(1,2 C_{\bar{\gamma}}, \bar{C}\right)$, by $(2-5)$ and $(2-6)$

$$
\begin{aligned}
& \|u-h\|_{L^{\infty}\left(\bar{B}_{8 / 9-\delta}\right)}+\|\xi\|_{L^{p}\left(B_{8 / 9-\delta}\right)} \\
& \leq \widehat{C}\|f\|_{L^{p}\left(B_{8 / 9-\delta}\right)}+\widehat{C}\left(\delta^{\bar{\gamma} / 2}+\epsilon_{1} \delta^{\frac{d-2 q}{q}+\frac{\bar{\gamma}}{2}-d}\right)\left(\|u\|_{L^{\infty}\left(B_{1}\right)}+\|f\|_{L^{p}\left(B_{1}\right)}\right) \\
& =\widehat{C} \delta^{\bar{\gamma} / 2}\left(1+\epsilon_{1} \delta^{\frac{d-2 q}{q}-d}\right)\|u\|_{L^{\infty}\left(B_{1}\right)} \\
& \quad+\widehat{C}\left(1+\delta^{\bar{\gamma} / 2}+\epsilon_{1} \delta^{\frac{d-2 q}{q}+\frac{\bar{\gamma}}{2}-d}\right)\|f\|_{L^{p}\left(B_{8 / 9-\delta}\right)} .
\end{aligned}
$$

If we take

$$
\delta=\epsilon_{1}^{-\left(\frac{d-2 q}{q}-d\right)^{-1}}=\epsilon_{1}^{\frac{q}{d q+2 q-d}} \quad \text { and } \quad \gamma=\frac{\bar{\gamma}}{2} \cdot \frac{q}{d q+2 q-d}
$$

then

$$
\begin{aligned}
\|u-h\|_{L^{\infty}\left(\bar{B}_{8 / 9-\delta}\right)}+\|\xi\|_{L^{p}\left(B_{8 / 9-\delta}\right)} & \\
& \leq 2 \widehat{C} \epsilon_{1}^{\gamma}\|u\|_{L^{\infty}\left(B_{1}\right)}+\widehat{C}\left(1+2 \epsilon_{1}^{\gamma}\right)\|f\|_{L^{p}\left(B_{8 / 9-\delta}\right)} \\
& \leq C_{1}\left(\epsilon_{1}^{\gamma}\|u\|_{L^{\infty}\left(B_{1}\right)}+\|f\|_{L^{p}\left(B_{8 / 9-\delta}\right)}\right) \\
& \leq C_{1}\left(\epsilon_{1}^{\gamma}\|u\|_{L^{\infty}\left(B_{1}\right)}+\|f\|_{L^{p}\left(B_{1}\right)}\right)
\end{aligned}
$$

where

$$
C_{1}=\max \left[2 \widehat{C} \sup _{x \in B_{1}}|u(x)|, \widehat{C}\left(1+2 \epsilon_{1}^{\gamma}\right)\right]
$$

Taking

$$
\delta<\frac{8}{9}-\frac{7}{8}=\frac{1}{72}
$$

we have $B_{7 / 8} \subset B_{8 / 9-\delta}$. Then we conclude

$$
\|u-h\|_{L^{\infty}\left(B_{7 / 8}\right)}+\|\xi\|_{L^{p}\left(B_{7 / 8}\right)} \leq C_{1}\left(\epsilon_{1}^{\gamma}\|u\|_{L^{\infty}\left(B_{1}\right)}+\|f\|_{L^{p}\left(B_{1}\right)}\right) .
$$

Lemma 2.2 Under the hypotheses of Lemma 2.1, suppose further that

$$
-\|x\|^{2} \leq u(x) \leq\|x\|^{2} \quad \text { in } B_{1} \backslash B_{6 / 7} .
$$

Then, if $\|u\|_{L^{\infty}\left(B_{1}\right)}<1$, there exist $\bar{M}>1$ and $\sigma \in(0,1)$ such that

$$
\left|A_{\bar{M}}\left(u, B_{1}\right) \cap Q_{1}\right| \leq \sigma
$$

Proof. If $d<p<q$, by Lemma 2.1 we can take $h \in W^{2, q}\left(B_{7 / 8}\right) \cap \mathcal{C}\left(\bar{B}_{8 / 9}\right)$ such that, for

$$
\xi=f-\sup _{\alpha \in \mathcal{A}} \inf _{\beta \in \mathcal{B}}\left[\operatorname{Tr}\left(A_{\alpha, \beta}(x) D^{2} h\right)\right]
$$




$$
\|u-h\|_{L^{\infty}\left(B_{7 / 8}\right)}+\|\xi\|_{L^{p}\left(B_{7 / 8}\right)}<C_{1}\left(\epsilon_{1}^{\gamma}+\|f\|_{L^{p}\left(B_{1}\right)}\right)
$$

where $C_{1}$ and $\gamma$ are non-negative constants depending on $(d, \lambda, \Lambda, C, q, p)$. Extend $h$ continuously to $B_{1}$ such that $h \equiv u$ in $B_{1} \backslash B_{8 / 9}$ and

$$
\|u-h\|_{L^{\infty}\left(B_{1}\right)}=\|u-h\|_{L^{\infty}\left(B_{7 / 8}\right)} .
$$

Note that, by the maximum principle

$$
\|h\|_{L^{\infty}\left(B_{7 / 8}\right)} \leq\|h\|_{L^{\infty}\left(\partial B_{8 / 9}\right)}=\|u\|_{L^{\infty}\left(\partial B_{8 / 9}\right)} \leq 1,
$$

assuming that $h$ is the solution of the Dirichlet problem (2-2). So, for every

$$
x \in B_{1} \backslash B_{8 / 9} \subset B_{1} \backslash B_{7 / 8} \subset B_{1} \backslash B_{6 / 7},
$$

it holds

$$
\begin{aligned}
|h(x)| & \leq|u(x)|+\|h-u\|_{L^{\infty}\left(B_{7 / 8}\right)} \\
& <\|x\|^{2}+\|h\|_{L^{\infty}\left(B_{7 / 8}\right)}+\|u\|_{L^{\infty}\left(B_{7 / 8}\right)} \\
& <\|x\|^{2}+2 .
\end{aligned}
$$

By combining the former inequality with the Lemma 1.13, we have

$$
\left|A_{t}\left(h, B_{1}\right) \cap Q_{1}\right| \leq C t^{-q}
$$

Next, if we define

$$
v=\frac{u-h}{2 C_{1} \epsilon_{1}^{\gamma}}
$$

then, by Lemma 1.8 and Theorem 1.9

$$
v \in S\left(\lambda, \Lambda, \frac{\xi(x)}{2 C_{1} \epsilon_{1}^{\gamma}}\right)
$$

hence, by the Lemma 1.16, there exist universal constants $C>0$ and $\mu>0$ such that, according to the definition of $A_{t}$,

$$
\left|A_{2 t C_{1} \epsilon_{1}^{\gamma}}\left(u-h, B_{1}\right) \cap Q_{1}\right|=\left|A_{t}\left(\frac{u-h}{2 C_{1} \epsilon_{1}^{\gamma}}, B_{1}\right) \cap Q_{1}\right| \leq C t^{-\mu},
$$

and

$$
\left|A_{t}\left(u-h, B_{1}\right) \cap Q_{1}\right| \leq C\left(\frac{t}{2 C_{1} \epsilon_{1}^{\gamma}}\right)^{-\mu}=C\left(2 C_{1}\right)^{\mu} \epsilon_{1}^{\gamma \mu} t^{-\mu} .
$$

According to the Definition $\mathbf{1 . 7}$, note that

$$
G_{t}\left(u-h, B_{1}\right) \cap G_{t}\left(h, B_{1}\right) \subset G_{2 t}\left(u, B_{1}\right) .
$$


Thus, taking complements on $B_{1}$

$$
A_{2 t}\left(u, B_{1}\right) \subset A_{t}\left(u-h, B_{1}\right) \cup A_{t}\left(h, B_{1}\right) .
$$

Finally,

$$
\begin{aligned}
\left|A_{2 t}\left(u, B_{1}\right) \cap Q\right| & \leq\left|A_{t}\left(u-h, B_{1}\right) \cap Q\right|+\left|A_{t}\left(h, B_{1}\right) \cap Q\right| \\
& \leq C\left(2 C_{1}\right)^{\mu} \epsilon_{1}^{\gamma \mu} t^{-\mu}+C t^{-q} .
\end{aligned}
$$

So, for the given $\sigma \in(0,1)$

$$
\left|A_{2 t}\left(u, B_{1}\right) \cap Q\right| \leq \sigma,
$$

when

$$
t \geq \max \left\{1,\left[C\left(2 C_{1}\right)^{\mu} \epsilon_{1}^{\gamma \mu} \cdot \frac{\sigma}{2}\right]^{-1 / \mu},\left[C \cdot \frac{\sigma}{2}\right]^{-1 / q}\right\} .
$$

Finally, take $\bar{M}=2 t>1$.

Lemma 2.3 Suppose the hypotheses of the Lemma 2.1 hold true and let $\bar{Q}$ be a cube such that $Q \subset \bar{Q}$. Then,

$$
G_{1}\left(u, B_{1}\right) \cap \bar{Q} \neq \emptyset \Longrightarrow\left|A_{M}\left(u, B_{1}\right) \cap Q\right| \leq \sigma,
$$

for some $M>1$.

Proof. Let $x_{0} \in G_{1}\left(u, B_{1}\right) \cap \bar{Q}$, then $x_{0} \in B_{1}$ and there exists an affine function $\ell$ such that

$$
|u(x)-\ell(x)| \leq \frac{\left\|x-x_{0}\right\|^{2}}{2} \leq C_{3}\|x\|^{2},
$$

for some $C_{3}>1$ and every $x \in B_{1}$. Notice that

$$
v:=\frac{u-\ell}{C_{3}} \Longrightarrow|v(x)| \leq\|x\|^{2}
$$

Then, for all $x \in B_{1} \backslash B_{6 / 7}$

$$
-\|x\|^{2} \leq v(x) \leq\|x\|^{2} \quad \text { in } B_{1} \backslash B_{6 / 7}
$$

Therefore, by the Lemma 2.2, there exists $\bar{M}>1$ such that

$$
\left|A_{C_{3} \bar{M}}\left(u, B_{1}\right) \cap Q\right|=\left|A_{\bar{M}}\left(v, B_{1}\right) \cap Q\right| \leq \sigma .
$$

At the end we can take $M=C_{3} \bar{M}$. 
Lemma 2.4 Under the hypotheses of Lemma 2.1, extend $f$ by zero outside of $B_{1}$ and set

$$
A:=A_{M^{k+1}}\left(u, B_{1}\right) \cap Q
$$

and

$$
B:=\left[A_{M^{k}}\left(u, B_{1}\right) \cap Q\right] \cup\left\{x \in Q \mid m\left(f^{p}\right) \geq\left(C M^{k}\right)^{p}\right\},
$$

where $C<\epsilon_{1} \cdot\left|B_{1}\right|^{-1 / p}$. Then,

$$
|A| \leq \sigma|B|
$$

for some $M>1$.

Proof. If there is a cube $\bar{Q}$ such that $Q \subset \bar{Q}$ and $G_{1}\left(u, B_{1}\right) \cap \bar{Q} \neq \emptyset$, Lemma 2.3 implies the existence of $\widetilde{M}>1$ such that

$$
|A|=\left|A_{M^{k+1}}\left(u, B_{1}\right) \cap Q\right| \leq\left|A_{M}\left(u, B_{1}\right) \cap Q\right| \leq\left|A_{\widetilde{M}}\left(u, B_{1}\right) \cap Q\right| \leq \sigma,
$$

for some $\sigma \in(0,1)$, an setting $M>\widetilde{M}$. Set the dyadic cube $K=Q_{1 / 2^{i}}$ and denote by $\bar{K}$ its predecessor. Next, notice that

$$
|A \cap K|>\sigma|K| \Longrightarrow \bar{K} \subset B
$$

Were (2-7) false, we would have

$$
|A \cap K|>\sigma|K| \text { and } \bar{K} \not \subset B .
$$

Then there would exists $x_{1} \in \bar{K}$, such that $x_{1} \notin A_{M^{k}}\left(u, B_{1}\right) \cap Q$ and

$$
\sup _{r>0} f_{B_{r}\left(x_{1}\right)} f^{p}(x) d x=m\left(f^{p}\right)\left(x_{1}\right)<\left(C M^{k}\right)^{p} \text {. }
$$

Let

$$
\bar{u}(y):=\frac{2^{2 i}}{M^{k}} u\left(x_{0}+\frac{y}{2^{i}}\right)
$$

where

$$
\left\|x_{0}-x_{1}\right\| \leq r-\frac{1}{2^{i}} \quad \text { and } \quad \frac{1}{2^{i}} \leq r<\frac{1}{2^{i}}\left(\frac{\epsilon_{1}}{C\left|B_{1}\right|^{1 / p}}\right)^{p / d} .
$$

Then $B_{2^{-i}}\left(x_{0}\right) \subset B_{r}\left(x_{1}\right)$ and

$$
D^{2} \bar{u}(y)=\frac{1}{M^{k}} D^{2} u\left(x_{0}+\frac{y}{2^{i}}\right) .
$$

Therefore we have that $\bar{u}$ is solution of

$$
\sup _{\alpha \in \mathcal{A}} \inf _{\beta \in \mathcal{B}}\left[-\operatorname{Tr}\left(A_{\alpha, \beta}(x) D^{2} \bar{u}(x)\right)\right]=\bar{f}(x),
$$


where

$$
\bar{f}(x):=\frac{1}{M^{k}} f\left(x_{0}+\frac{x}{2^{i}}\right) .
$$

We also have that

$$
\begin{aligned}
\|\bar{f}\|_{L^{p}\left(B_{1}\right)}^{p} & =\frac{1}{M^{p k}} \int_{B_{1}}\left|f\left(x_{0}+\frac{y}{2^{i}}\right)\right|^{p} d y \\
& =\frac{2^{i d}}{M^{p k}} \int_{B_{2^{-i}}\left(x_{0}\right)}|f(x)|^{p} d x \\
& \leq \frac{2^{i d}}{M^{p k}} \int_{B_{r}\left(x_{1}\right)}|f(x)|^{p} d x \\
& <\frac{2^{i d}}{M^{p k}}\left(C M^{k}\right)^{p}\left|B_{r}\left(x_{1}\right)\right| \\
& =\left(2^{i} r\right)^{d} C^{p}\left|B_{1}\right| \\
& \leq \epsilon_{1}^{p} .
\end{aligned}
$$

Note that

$$
G_{M^{k+1}}\left(u, B_{1}\right)=G_{M}\left(\frac{u}{M^{k}}, B_{1}\right)=2^{-i} G_{M}\left(\bar{u}, B_{2^{-i}}\left(x_{0}\right)\right),
$$

which implies

$$
G_{1}\left(\bar{u}, B_{2^{-i}}\left(x_{0}\right)\right) \cap \bar{Q}=2^{i} G_{1}\left(u, B_{1}\right) \cap \bar{Q} \neq \emptyset .
$$

So, by Lemma 2.3 ,

$$
\frac{\left|A_{M^{k+1}}\left(u, B_{1}\right) \cap Q\right|}{|K|}=\left|A_{M}\left(\bar{u}, B_{2^{-i}}\left(x_{0}\right)\right) \cap Q\right| \leq \sigma,
$$

since $|K|=2^{-i d}$. Then

$$
|A \cap K| \leq\left|A_{M^{k+1}}\left(u, B_{1}\right) \cap Q\right| \leq \sigma \cdot|K| .
$$

This is a contradiction with the assumption

$$
|A \cap K|>\sigma|K|
$$

Finally, because (2-7) holds true, Lemma 1.14 leads to

$$
|A| \leq \sigma|B|
$$




\subsection{1}

\section{Sobolev Regularity}

In what follows, we detail the proof of the main theorem in this chapter, namely, the Sobolev regularity for the solutions to the Isaacs equation.

Theorem 2.5 Let $u \in \mathcal{C}\left(B_{1}\right)$ be a viscosity solution to

$$
\sup _{\alpha \in \mathcal{A}} \inf _{\beta \in \mathcal{B}}\left[-\operatorname{Tr}\left(A_{\alpha, \beta}(x) D^{2} u\right)\right]=f(x) \quad \text { in } \quad B_{1} \text {. }
$$

Under the hypotheses of the Lemma 2.1, $u \in W_{\text {loc }}^{2, p}\left(B_{1}\right)$ and

$$
\|u\|_{W^{2, p}\left(B_{1 / 2}\right)} \leq C\left(\|u\|_{L^{\infty}\left(B_{1}\right)}+\|f\|_{L^{p}\left(B_{1}\right)}\right)
$$

where $C>0$ is a universal constant.

Proof. By the Lemma 1.11, we have that

$$
\Theta\left(u, B_{1 / 2}\right) \in L^{p}\left(B_{1 / 2}\right) \Longrightarrow u \in W^{2, p}\left(B_{1 / 2}\right) .
$$

Because of Lemma 1.15,

$$
\Theta\left(u, B_{1 / 2}\right) \in L^{p}\left(B_{1 / 2}\right) \Longleftrightarrow \sum_{k=1}^{\infty} M^{p k} \mu_{\Theta}\left(M^{k}\right)<\infty
$$

where

$$
\mu_{\Theta}(t):=\left|\left\{x \in B_{1 / 2} \mid \Theta\left(u, B_{1 / 2}\right)(x)>t\right\}\right|
$$

Since

$$
\mu_{\Theta}(t) \leq\left|A_{t}\left(u, B_{1 / 2}\right)\right|,
$$

we have that $u \in W^{2, p}\left(B_{1 / 2}\right)$ whenever

$$
\sum_{k=1}^{\infty} M^{p k}\left|A_{M^{k}}\left(u, B_{1 / 2}\right)\right|<\infty .
$$

Now we prove (2-8); set

$$
a_{k}:=\left|A_{M^{k}}\left(u, B_{1}\right) \cap Q_{1}\right|
$$

and

$$
b_{k}:=\left|\left\{x \in Q_{1} \mid m\left(f^{p}\right)(x) \geq\left(C M^{k}\right)^{p}\right\}\right| .
$$

Then Lemma 2.4 leads to

$$
a_{k+1} \leq \sigma\left(a_{k}+b_{k}\right)
$$


for some $M>1$ and $\sigma \in(0,1)$. Next, for all $k \geq 1$,

$$
\begin{aligned}
a_{k} \leq & \sigma a_{k-1}+\sigma b_{k-1} \\
& \leq \sigma^{2} a_{k-2}+\sigma^{2} b_{k-2}+\sigma b_{k-1} \\
& \vdots \\
& \leq \sigma^{k} a_{0}+\sigma^{k} b_{0}+\sigma^{k-1} b_{1}+\cdots+\sigma^{2} b_{k-2}+\sigma b_{k-1} .
\end{aligned}
$$

Thus we obtain

$$
a_{k} \leq \sigma^{k} a_{0}+\sum_{i=0}^{k-1} \sigma^{k-i} b_{i} \leq \sigma^{k}+\sum_{i=0}^{k-1} \sigma^{k-i} b_{i} .
$$

Setting

$$
g(x):=\left[m\left(f^{p}\right)(x)\right]^{1 / p}
$$

we have

$$
b_{k}=\left|\left\{x \in Q_{1} \mid\left[m\left(f^{p}\right)\right]^{1 / p} \geq C M^{k}\right\}\right|=\mu_{g}\left(C M^{k}\right) .
$$

Since $f \in L^{p}\left(B_{1}\right)$, by the properties of the maximal functions,

$$
m\left(f^{p}\right) \in L^{1}\left(B_{1 / 2}\right) \Longrightarrow\left[m\left(f^{p}\right)\right]^{1 / p} \in L^{p}\left(B_{1 / 2}\right) .
$$

Thus, by Lemma 1.15,

$$
\sum_{k=0}^{\infty} M^{p k} b_{k}=\sum_{k=0}^{\infty} M^{p k} \mu_{g}\left(C M^{k}\right)<\infty .
$$

Finally, by taking $\left(\sigma M^{p}\right)<1$, we have:

$$
\begin{aligned}
\sum_{k=1}^{\infty} M^{p k} a_{k} & \leq \sum_{k=1}^{\infty} M^{p k}\left(\sigma^{k}+\sum_{i=0}^{k-1} \sigma^{k-i} b_{i}\right) \\
& =\sum_{k=1}^{\infty}\left(\sigma M^{p}\right)^{k}+\sum_{k=1}^{\infty} \sum_{i=0}^{k-1}\left(\sigma M^{p}\right)^{k-i} M^{p i} b_{i} \\
& \leq \sum_{k=1}^{\infty}\left(\sigma M^{p}\right)^{k}+\left(\sum_{j=1}^{\infty}\left(\sigma M^{p}\right)^{j}\right)\left(\sum_{i=0}^{\infty} M^{p i} b_{i}\right) \\
& <\infty .
\end{aligned}
$$

Noting that $B_{1 / 2} \subset Q_{1}$,

$$
\left|A_{M^{k}}\left(u, B_{1 / 2}\right)\right| \leq\left|A_{M^{k}}\left(u, B_{1}\right) \cap Q_{1}\right|=a_{k},
$$

which implies (2-8). Then $u \in W^{2, p}\left(B_{1 / 2}\right)$ and the proof is complete. 


\section{2}

\section{Second Approximation Lemma}

In this section we include variants of the Isaacs equation with explicit dependence on lower order terms. To that end, we start with a second approximation result.

Lemma 2.6 (Second Approximation Lemma) Let $u \in \mathcal{C}\left(B_{1}\right)$ be a viscosity solution to

$$
\sup _{\alpha \in \mathcal{A}} \inf _{\beta \in \mathcal{B}}\left[-\operatorname{Tr}\left(A_{\alpha, \beta}(x) D^{2} u\right)-\mathbf{b}_{\alpha, \beta}(x) \cdot D u\right]=f(x) \quad \text { in } \quad B_{1}
$$

where $A_{\alpha, \beta}: B_{1} \times \mathcal{A} \times \mathcal{B} \rightarrow \mathbb{R}^{d^{2}}$ is a $(\lambda, \Lambda)$-elliptic matrix and $\mathbf{b}_{\alpha, \beta}:$ $B_{1} \times \mathcal{A} \times \mathcal{B} \rightarrow \mathbb{R}^{d}$ is a vector field such that $\mathbf{b}_{\alpha, \beta} \in L^{p}\left(B_{1}\right)$ uniformly. That is

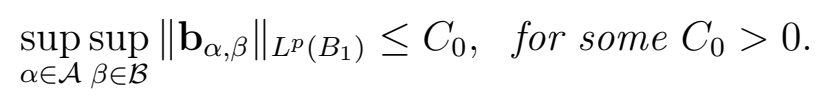

For every $\delta>0$ it is possible to choose $\epsilon_{1}>0$ such that, if $\bar{A}_{\beta}: B_{1} \times \mathcal{B} \rightarrow \mathbb{R}^{d^{2}}$ satisfies

$$
\|f\|_{L^{p}\left(B_{1}\right)}+\left\|A_{\alpha, \beta}-\bar{A}_{\beta}\right\|_{L^{\infty}\left(B_{1}\right)}<\epsilon_{1},
$$

there exists $h \in W_{\mathrm{loc}}^{2, q}\left(B_{1}\right)$ satisfying

$$
\begin{cases}\inf _{\beta \in \mathcal{B}}\left[-\operatorname{Tr}\left(\bar{A}_{\beta}(x) D^{2} h\right)\right]=0 & \text { in } \quad B_{8 / 9} \\ h=u & \text { on } \quad \partial B_{8 / 9}\end{cases}
$$

with

$$
\|h\|_{W^{2, p}\left(B_{1 / 2}\right)} \leq C \quad \text { and } \quad\|u-h\|_{L^{\infty}\left(B_{7 / 8}\right)} \leq \delta,
$$

for some universal constant $C>0$.

Proof. Suppose by contradiction, that the statement is false. In that case, we can take sequences $\left(A_{\alpha, \beta}^{n}\right)_{n \in \mathbb{N}},\left(\mathbf{b}_{\alpha, \beta}^{n}\right)_{n \in \mathbb{N}}$, such that $A_{\alpha, \beta}^{n}: B_{1} \times \mathcal{A} \times \mathcal{B} \rightarrow \mathbb{R}^{d^{2}}$ are $(\lambda, \Lambda)$-elliptic matrices and $\mathbf{b}_{\alpha, \beta}^{n}: B_{1} \times \mathcal{A} \times \mathcal{B} \rightarrow \mathbb{R}^{d}$ are vector fields such that $\mathbf{b}_{\alpha, \beta}^{n} \in L^{p}\left(B_{1}\right)$ uniformly, and sequences of functions $\left(u_{n}\right)_{n \in \mathbb{N}}$ and $\left(f_{n}\right)_{n \in \mathbb{N}}$ satisfying

$$
\sup _{\alpha \in \mathcal{A}} \inf _{\beta \in \mathcal{B}}\left[-\operatorname{Tr}\left(A_{\alpha, \beta}^{n}(x) D^{2} u_{n}\right)-\mathbf{b}_{\alpha, \beta}^{n}(x) \cdot D u_{n}\right]=f_{n}(x) \quad \text { in } \quad B_{1},
$$

with

$$
\left|A_{\alpha, \beta}^{n}-\bar{A}_{\beta}\right|+\left\|\mathbf{b}_{\alpha, \beta}^{n}\right\|_{L^{p}\left(B_{1}\right)}+\left\|f_{n}\right\|_{L^{p}\left(B_{1}\right)}<\frac{1}{n} .
$$


Moreover, there exists $\delta_{0}>0$ such that, for every solution $h$ to

$$
\begin{cases}\inf _{\beta \in \mathcal{B}}\left[-\operatorname{Tr}\left(\bar{A}_{\beta}(x) D^{2} h\right)\right]=0 & \text { in } \quad B_{8 / 9} \\ h=u_{n} & \text { on } \quad \partial B_{8 / 9}\end{cases}
$$

we obtain

$$
\left\|u_{n}-h\right\|_{L^{\infty}\left(B_{7 / 8}\right)}>\delta_{0}
$$

for any $n \in \mathbb{N}$.

By the assumptions, we have that $A_{\alpha, \beta}^{n} \rightarrow \bar{A}_{\beta}$ and $f_{n} \rightarrow 0$ when $n \rightarrow+\infty$, in the appropriate spaces. Moreover, by the regularity theory available for $u_{n}$, it converges to a function $u_{\infty}$ (through a subsequence if necessary). By the stability of the viscosity solutions, we have

$$
\inf _{\beta \in \mathcal{B}}\left[-\operatorname{Tr}\left(\bar{A}_{\beta}(x) D^{2} u_{\infty}\right)\right]=0 \quad \text { in } \quad B_{1} .
$$

Notice that by Theorem 1.17, $u_{\infty} \in W^{2, q}\left(B_{7 / 8}\right) \cap \mathcal{C}\left(B_{8 / 9}\right)$ with the appropriate estimates. By taking $h=u_{\infty}$ we obtain a contradiction.

\subsection{1}

\section{Sobolev Regularity}

Theorem 2.7 (Estimates in $\left.W^{1, \bar{p}}\right) \quad$ Let $u \in \mathcal{C}\left(B_{1}\right)$ be a viscosity solution to

$$
\sup _{\alpha \in \mathcal{A}} \inf _{\beta \in \mathcal{B}}\left[-\operatorname{Tr}\left(A_{\alpha, \beta}(x) D^{2} u\right)-\mathbf{b}_{\alpha, \beta}(x) \cdot D u\right]=f(x) \quad \text { in } \quad B_{1}
$$

and suppose the hypotheses of the Lemma 2.6 are in force. Then we have $u \in W_{\mathrm{loc}}^{1, \bar{p}}\left(B_{1}\right)$ and there exists $C>0$ such that

$$
\|u\|_{W^{1, \bar{p}}\left(B_{1 / 2}\right)} \leq C\left(\|u\|_{L^{\infty}\left(B_{1}\right)}+\|f\|_{L^{p}\left(B_{1}\right)}\right)
$$

for every $\bar{p} \in(1, \infty)$.

Proof. Follows from [(S97), Theorem 2.1].

Theorem 2.8 (Estimates in $W^{2, p}$ ) Let $u \in \mathcal{C}\left(B_{1}\right)$ be a viscosity solution to

$$
\sup _{\alpha \in \mathcal{A}} \inf _{\beta \in \mathcal{B}}\left[-\operatorname{Tr}\left(A_{\alpha, \beta}(x) D^{2} u\right)-\mathbf{b}_{\alpha, \beta}(x) \cdot D u\right]=f(x) \quad \text { in } \quad B_{1}
$$

and suppose the hypotheses of the Lemma 2.6 are in force. Then $u \in W_{\mathrm{loc}}^{2, p}\left(B_{1}\right)$ and

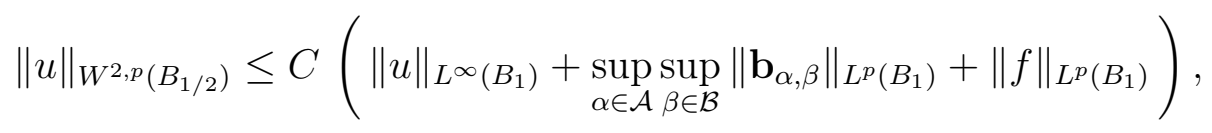


where $C>0$ is a universal constant.

Proof. Let

$$
g(x):=\sup _{\alpha \in \mathcal{A}} \inf _{\beta \in \mathcal{B}}\left[-\operatorname{Tr}\left(A_{\alpha, \beta}(x) D^{2} u(x)\right)\right] .
$$

Because $u$ satisfies

$$
\sup _{\alpha \in \mathcal{A}} \inf _{\beta \in \mathcal{B}}\left[-\operatorname{Tr}\left(A_{\alpha, \beta}(x) D^{2} u\right)-\mathbf{b}_{\alpha, \beta}(x) \cdot D u\right]=f(x) \quad \text { in } \quad B_{1}
$$

we have that, for every $x \in B_{1}$

$$
\begin{aligned}
|g(x)| & =\left|\sup _{\alpha \in \mathcal{A}} \inf _{\beta \in \mathcal{B}}\left[-\operatorname{Tr}\left(A_{\alpha, \beta}(x) D^{2} u(x)\right)\right]\right| \\
\leq & \left|\sup _{\alpha \in \mathcal{A}} \sup _{\beta \in \mathcal{B}} \mathbf{b}_{\alpha, \beta}(x) \cdot D u(x)\right| \\
& \quad+\left|\sup _{\alpha \in \mathcal{A}} \inf _{\beta \in \mathcal{B}}\left[-\operatorname{Tr}\left(A_{\alpha, \beta}(x) D^{2} u(x)\right)-\mathbf{b}_{\alpha, \beta}(x) \cdot D u(x)\right]\right| \\
& =\sup _{\alpha \in \mathcal{A}} \sup _{\beta \in \mathcal{B}}\left|\mathbf{b}_{\alpha, \beta}(x) \cdot D u(x)\right|+|f(x)| \\
\leq & C_{0} \sup _{\alpha \in \mathcal{A}} \sup _{\beta \in \mathcal{B}}\left|\mathbf{b}_{\alpha, \beta}(x)\right| \cdot\|D u\|+|f(x)|,
\end{aligned}
$$

for some universal constant $C_{0}>0$. Thus $g \in L^{p}\left(B_{1}\right)$, because of Theorem 2.7. Note that $u$ is also a viscosity solution to

$$
\sup _{\alpha \in \mathcal{A}} \inf _{\beta \in \mathcal{B}}\left[-\operatorname{Tr}\left(A_{\alpha, \beta}(x) D^{2} u(x)\right)\right]=g(x) \quad \text { in } \quad B_{1} .
$$

Then, by Theorem 2.5, $u \in W_{\text {loc }}^{2, p}\left(B_{1}\right)$ and

$$
\begin{aligned}
& \|u\|_{W^{2, p}\left(B_{1 / 2}\right)} \leq \widetilde{C}\left(\|u\|_{L^{\infty}\left(B_{1}\right)}+\|g\|_{L^{p}\left(B_{1}\right)}\right) \\
& \leq C\left(\|u\|_{L^{\infty}\left(B_{1}\right)}+\sup _{\alpha \in \mathcal{A}} \sup _{\beta \in \mathcal{B}}\left\|\mathbf{b}_{\alpha, \beta}\right\|_{L^{p}\left(B_{1}\right)}+\|f\|_{L^{p}\left(B_{1}\right)}\right) .
\end{aligned}
$$




\section{Estimates in Hölder Spaces}

In this last chapter we examine the Hölder regularity of the Isaacs equation. We begin with another approximation Lemma. Then, we establish $\mathcal{C}_{\text {loc }}^{1, \text { Log-Lip }}$-regularity in $B_{1}$, which implies $\mathcal{C}_{\text {loc }}^{1, \gamma}$-regularity for every $\gamma \in(0,1)$. To conclude, under suitable conditions, we proof $C_{\mathrm{loc}}^{2, \gamma}$-regularity for the solutions.

\section{1}

\section{Approximation Lemma}

We open the chapter with another approximation lemma.

Lemma 3.1 (Third Approximation Lemma) Let $u \in \mathcal{C}\left(B_{1}\right)$ be a viscosity solution of

$$
\sup _{\alpha \in \mathcal{A}} \inf _{\beta \in \mathcal{B}}\left[-\operatorname{Tr}\left(A_{\alpha, \beta}(x)\left(D^{2} u+M\right)\right)+c\right]=f(x) \quad \text { in } \quad B_{1},
$$

where $A_{\alpha, \beta}: B_{1} \times \mathcal{A} \times \mathcal{B} \rightarrow \mathbb{R}^{d^{2}}$ is a $(\lambda, \Lambda)$-elliptic matrix, $c \in \mathbb{R}$ and $M \in \mathcal{S}(d)$ satisfies

$$
\inf _{\beta \in \mathcal{B}}\left[-\operatorname{Tr}\left(\bar{A}_{\beta}(0) M\right)+c\right]=0 .
$$

For every $\delta>0$ it is possible to choose $\epsilon_{2}>0$ such that, if $\bar{A}_{\beta}: B_{1} \times \mathcal{B} \rightarrow \mathbb{R}^{d^{2}}$ satisfies

$$
\|f\|_{L^{p}\left(B_{1}\right)}+\sup _{x \in B_{1}}\left|A_{\alpha, \beta}(x)-\bar{A}_{\beta}(0)\right|<\epsilon_{2},
$$

there exists $h \in \mathcal{C}_{\text {loc }}^{2, \gamma}\left(B_{1}\right)$ solution to

$$
\begin{cases}\inf _{\beta \in \mathcal{B}}\left[-\operatorname{Tr}\left(\bar{A}_{\beta}(0)\left(D^{2} h+M\right)\right)+c\right]=0 & \text { in } \quad B_{8 / 9} \\ h=u & \text { on } \quad \partial B_{8 / 9}\end{cases}
$$

such that

$$
\|h\|_{\mathcal{C}_{\text {loc }}^{2, \gamma\left(B_{1}\right)}} \leq C \quad \text { and } \quad\|u-h\|_{L^{\infty}\left(B_{7 / 8}\right)} \leq \delta
$$

for some $C>0$ and $\gamma \in(0,1)$, universal constants.

Proof. Suppose by contradiction, that the statement is false. In that case, we can take a sequence $\left(A_{\alpha, \beta}^{n}\right)_{n \in \mathbb{N}}$ such that $A_{\alpha, \beta}^{n}: B_{1} \times \mathcal{A} \times \mathcal{B} \rightarrow \mathbb{R}^{d^{2}}$ are $(\lambda, \Lambda)$ - 
elliptic matrices, and sequences of functions $\left(u_{n}\right)_{n \in \mathbb{N}}$ and $\left(f_{n}\right)_{n \in \mathbb{N}}$ satisfying

$$
\sup _{\alpha \in \mathcal{A}} \inf _{\beta \in \mathcal{B}}\left[-\operatorname{Tr}\left(A_{\alpha, \beta}^{n}(x)\left(D^{2} u_{n}+M\right)\right)+c\right]=f_{n}(x) \quad \text { in } \quad B_{1}
$$

with

$$
\sup _{x \in B_{1}}\left|A_{\alpha, \beta}^{n}(x)-\bar{A}_{\beta}(0)\right|+\left\|f_{n}\right\|_{L^{p}\left(B_{1}\right)}<\frac{1}{n},
$$

and there is $\delta_{0}>0$ such that, for every solution $h$ to

$$
\left\{\begin{array}{lr}
\inf _{\beta \in \mathcal{B}}\left[-\operatorname{Tr}\left(\bar{A}_{\beta}(0)\left(D^{2} h+M\right)\right)+c\right]=0 & \text { in } \quad B_{8 / 9}, \\
h=u_{n} & \text { on } \quad \partial B_{8 / 9},
\end{array}\right.
$$

we obtain

$$
\left\|u_{n}-h\right\|_{L^{\infty}\left(B_{7 / 8}\right)}>\delta_{0}, \quad \forall n \in \mathbb{N}
$$

By the assumptions made, we have that $A_{\alpha, \beta}^{n} \rightarrow \bar{A}_{\beta}(0)$ uniformly and $f_{n} \rightarrow 0$ in the appropriate topology, when $n \rightarrow+\infty$. Moreover, by the regularity theory available for $u_{n}$, it converges to a function $u_{\infty}$ (through a subsequence if necessary). By the stability of the viscosity solutions, we infer that $u_{\infty}$ solves the Bellman equation

$$
\inf _{\beta \in \mathcal{B}}\left[-\operatorname{Tr}\left(\bar{A}_{\beta}(0)\left(D^{2} u_{\infty}+M\right)\right)+c\right]=0 \quad \text { in } \quad B_{8 / 9}
$$

so by the Theorem 1.3, $u_{\infty} \in C^{2, \gamma}\left(\bar{B}_{1 / 2}\right)$, for some $\gamma \in(0,1)$. By taking $h=u_{\infty}$ we obtain a contradiction.

\section{2}

\section{Estimates in $\mathcal{C}_{\text {loc }}^{1, \log -\operatorname{Lip}}\left(B_{1}\right)$}

In this section we establish the regularity of the solutions to the Isaacs equation in $\mathcal{C}^{1, \log -\text { Lip }}$-spaces. We start with a definition.

Definition 3.1 (Log-Lipschitz Continuity) A function $u: B_{1} \rightarrow \mathbb{R}$ is called Log-Lipschitz continuous if there exists a constant $C>0$ such that

$$
|u(x)-u(y)| \leq C\|x-y\| \log \left(\frac{1}{\|x-y\|}\right)
$$

where

$$
0<\|x-y\| \ll 1
$$

The set of Log-Lipschitz continuous functions defined on the open set $\mathcal{O}$ is denoted $\mathcal{C}^{\log -\operatorname{Lip}}(\mathcal{O})$. 
We observe that a log-Lipschitz continuous function is $\gamma$-Hölder continuous for every $\gamma \in(0,1)$. In fact, notice that

$$
\|x\| \leq\|x\| \log \left(\frac{1}{\|x\|}\right) \leq(1-\gamma)^{-1}\|x\|^{\gamma}
$$

for any $\gamma \in(0,1)$. Therefore, we have

$$
\mathcal{C}_{\text {loc }}^{0,1}\left(B_{1}\right) \subset \mathcal{C}_{\text {loc }}^{\text {Log-Lip }}\left(B_{1}\right) \subset \mathcal{C}_{\text {loc }}^{0, \gamma}\left(B_{1}\right)
$$

Moreover, we say that $u \in \mathcal{C}_{\text {loc }}^{1, \log -\operatorname{Lip}}\left(B_{1}\right)$ if $D u \in C_{\mathrm{loc}}^{\mathrm{Log}-\operatorname{Lip}}\left(B_{1}\right)$, and it is equivalent to say that there exist $\rho \in(0,1)$ and $C>0$ such that

$$
\sup _{\left\|x-x_{0}\right\|<\rho}\left|u(x)-u\left(x_{0}\right)-D u\left(x_{0}\right) \cdot\left(x-x_{0}\right)\right| \leq C \rho^{2} \log \left(\frac{1}{\rho}\right) .
$$

for any $x_{0} \in B_{1}$.

In order to proof the Theorem $3.4\left(\mathcal{C}_{\text {loc }}^{1, \text { Log-Lip }}\right.$ regularity), we establish and proof an iteration result (Lemma 3.3). We first proof the inductive step of the Lemma $\mathbf{3 . 3}$ as follows.

Lemma 3.2 Let $u \in \mathcal{C}\left(B_{1}\right)$ be a viscosity solution of

$$
\sup _{\alpha \in \mathcal{A}} \inf _{\beta \in \mathcal{B}}\left[-\operatorname{Tr}\left(A_{\alpha, \beta}(x) D^{2} u\right)\right]=f(x) \quad \text { in } \quad B_{1} \text {, }
$$

where $A_{\alpha, \beta}: B_{1} \times \mathcal{A} \times \mathcal{B} \rightarrow \mathbb{R}^{d^{2}}$ is a $(\lambda, \Lambda)$-elliptic matrix. Suppose that, for some $n \in \mathbb{N}$ there exist $0<\rho \ll 1$ and a finite sequence of polynomials $\left(P_{k}\right)_{k \leq n}$, of the form

$$
P_{k}(x):=a_{k}+\mathbf{b}_{k} \cdot x+\frac{1}{2} x^{T} C_{k} x
$$

satisfying

$$
\inf _{\beta \in \mathcal{B}}\left[-\operatorname{Tr}\left(\bar{A}_{\beta}(0) C_{k}\right)\right]=\langle f\rangle \quad \text { and } \quad\left\|u-P_{k}\right\|_{L^{\infty}\left(B_{\rho^{k}}\right)} \leq \rho^{2 k}
$$

for some $\bar{A}_{\beta}: B_{1} \times \mathcal{B} \rightarrow \mathbb{R}^{d^{2}}$, a $(\lambda, \Lambda)$-elliptic matrix. Moreover, suppose that for every $k \leq n$, we have

$$
\left|a_{k}-a_{k-1}\right|+\rho^{k-1}\left\|\mathbf{b}_{k}-\mathbf{b}_{k-1}\right\|+\rho^{2(k-1)}\left\|C_{k}-C_{k-1}\right\| \leq C \rho^{2(k-1)} .
$$

Let $v_{n}: B_{1} \rightarrow \mathbb{R}$ be defined by

$$
v_{n}(x):=\frac{\left(u-P_{n}\right)\left(\rho^{n} x\right)}{\rho^{2 n}} .
$$


Then for every $\delta>0$ and $\gamma \in(0,1)$, there exist $\epsilon_{2}>0$ and $h \in \mathcal{C}_{\text {loc }}^{2, \gamma}\left(B_{1}\right)$ the solution of

$$
\begin{cases}\inf _{\beta \in \mathcal{B}}\left[-\operatorname{Tr}\left(\bar{A}_{\beta}(0)\left(D^{2} h+C_{n}\right)\right)\right]=\langle f\rangle & \text { in } \quad B_{8 / 9}, \\ h=v_{n} & \text { on } \quad \partial B_{8 / 9},\end{cases}
$$

such that, if

$$
\sup _{r \in(0,1]} f_{B_{r}}|f(x)-\langle f\rangle|^{p} d x \leq \epsilon_{2}^{p} \quad \text { and } \quad \sup _{x \in B_{1}}\left|A_{\alpha, \beta}(x)-\bar{A}_{\beta}(0)\right|<\epsilon_{2},
$$

uniformly in $\alpha$ and $\beta$, then

$$
\|h\|_{\mathcal{C}_{\text {loc }}^{2, \gamma}\left(B_{1}\right)} \leq C \quad \text { and } \quad\left\|v_{n}-h\right\|_{L^{\infty}\left(B_{7 / 8}\right)} \leq \delta
$$

for some $C>0$ universal constant.

Proof. We have that

$$
D^{2} v_{n}(x)=\frac{\rho^{2 n} D^{2} u\left(\rho^{n} x\right)-\rho^{2 n} C_{n}}{\rho^{2 n}}=D^{2} u\left(\rho^{n} x\right)-C_{n},
$$

and, for all $x \in B_{1}$,

$$
\sup _{\alpha \in \mathcal{A}} \inf _{\beta \in \mathcal{B}}\left[-\operatorname{Tr}\left(A_{\alpha, \beta}\left(\rho^{n} x\right)\left(D^{2} v_{n}(x)+C_{n}\right)\right)\right]=f\left(\rho^{n} x\right) .
$$

By taking $f_{n}(x):=f\left(\rho^{n} x\right)$, we get that $v_{n}$ solves

$$
\sup _{\alpha \in \mathcal{A}} \inf _{\beta \in \mathcal{B}}\left[-\operatorname{Tr}\left(A_{\alpha, \beta}\left(\rho^{n} x\right)\left(D^{2} v_{n}+C_{n}\right)\right)\right]=f_{n}(x) \quad \text { in } \quad B_{1} .
$$

Next, $v_{n}$ solves

$$
\sup _{\alpha \in \mathcal{A}} \inf _{\beta \in \mathcal{B}}\left[-\operatorname{Tr}\left(A_{\alpha, \beta}\left(\rho^{n} x\right)\left(D^{2} v_{n}+C_{n}\right)\right)-\langle f\rangle\right]=f_{n}(x)-\langle f\rangle \quad \text { in } \quad B_{1} \text {, }
$$

Noting that $\langle f\rangle=\left\langle f_{n}\right\rangle=\langle f\rangle_{\rho^{n}}$, we have that

$$
\begin{aligned}
\left\|f_{n}-\left\langle f_{n}\right\rangle\right\|_{L^{p}\left(B_{1}\right)}^{p} & =\left\|f_{n}-\left\langle f_{n}\right\rangle\right\|_{L^{p}\left(B_{1}\right)}^{p} \\
& =\frac{1}{\rho^{n d}} \int_{B_{\rho^{n}}}\left|f(x)-\langle f\rangle_{\rho^{n}}\right|^{p} d x \\
& \leq \sup _{r \in(0,1]} f_{B_{r}}\left|f(x)-\langle f\rangle_{r}\right|^{p} d x \\
& \leq \epsilon_{2}^{p} .
\end{aligned}
$$


Finally, by Lemma 3.1, for any $\delta>0$, there exists $h \in \mathcal{C}_{\text {loc }}^{2, \gamma}\left(B_{1}\right)$ solving

$$
\begin{cases}\inf _{\beta \in \mathcal{B}}\left[-\operatorname{Tr}\left(\bar{A}_{\beta}(0)\left(D^{2} h+C_{n}\right)\right)-\langle f\rangle\right]=0 & \text { in } \quad B_{8 / 9}, \\ h=v_{n} & \text { on } \quad \partial B_{8 / 9}\end{cases}
$$

such that

$$
\left\|v_{n}-h\right\|_{L^{\infty}\left(B_{1 / 2}\right)} \leq \delta
$$

where $\gamma \in(0,1)$ is a universal constant, and since $\langle f\rangle$ is constant, we obtain

$$
\inf _{\beta \in \mathcal{B}}\left[-\operatorname{Tr}\left(\bar{A}_{\beta}(0)\left(D^{2} h+C_{n}\right)\right)\right]=\langle f\rangle
$$

and complete the argument.

Lemma 3.3 Let $u \in \mathcal{C}\left(B_{1}\right)$ be a viscosity solution of

$$
\sup _{\alpha \in \mathcal{A}} \inf _{\beta \in \mathcal{B}}\left[-\operatorname{Tr}\left(A_{\alpha, \beta}(x) D^{2} u\right)\right]=f(x) \quad \text { in } \quad B_{1}
$$

where $A_{\alpha, \beta}: B_{1} \times \mathcal{A} \times \mathcal{B} \rightarrow \mathbb{R}^{d^{2}}$ is a $(\lambda, \Lambda)$-elliptic matrix and $\|u\|_{\mathcal{C}\left(\bar{B}_{1}\right)} \leq 1 / 2$. Then there exist constants $C>0, \epsilon_{2}>0$ and $0<\rho \ll 1$, and a sequence of polynomials $\left(P_{n}\right)_{n \in \mathbb{N}}$ given by

$$
P_{n}(x):=a_{n}+\mathbf{b}_{n} \cdot x+\frac{1}{2} x^{T} C_{n} x
$$

such that, if

$$
\sup _{r \in\left(0, r_{0}\right]} f_{B_{r}\left(x_{0}\right)}\left|f(x)-\langle f\rangle_{r_{0}, x_{0}}\right|^{p} d x \leq \epsilon_{2}^{p} \quad \text { and } \quad \sup _{x \in B_{r_{0}}}\left|A_{\alpha, \beta}(x)-\bar{A}_{\beta}\left(x_{0}\right)\right|<\epsilon_{2}
$$

for every $x_{0} \in B_{1}$ and $(\alpha, \beta) \in \mathcal{A} \times \mathcal{B}$, with $r_{0}=\operatorname{dist}\left(x_{0}, \partial B_{1}\right)$, then

$$
\inf _{\beta \in \mathcal{B}}\left[-\operatorname{Tr}\left(\bar{A}_{\beta}\left(x_{0}\right) C_{n}\right)\right]=\langle f\rangle, \quad \sup _{x \in B_{\rho^{n}}}\left|u(x)-P_{n}(x)\right| \leq \rho^{2 n}
$$

and

$$
\left|a_{n}-a_{n-1}\right|+\rho^{n-1}\left\|\mathbf{b}_{n}-\mathbf{b}_{n-1}\right\|+\rho^{2(n-1)}\left\|C_{n}-C_{n-1}\right\| \leq C \rho^{2(n-1)},
$$

for every $n \geq 0$.

Proof. We proceed by induction in $n \in \mathbb{N}$ and consider the case $x_{0}=0$.

In the basic case, take $P_{-1}=P_{0}=\frac{1}{2} x^{T} C_{0} x$ for $C_{0} \in S(d)$ such that

$$
\inf _{\beta \in \mathcal{B}}\left[-\operatorname{Tr}\left(\bar{A}_{\beta}(0) C_{0}\right)\right]=\langle f\rangle \quad \text { and } \quad\left\|C_{0}\right\| \leq 1
$$


Hence,

$$
a_{-1}=a_{0}=0 \quad, \mathbf{b}_{-1}=\mathbf{b}_{0}=0 \quad \text { and } \quad C_{-1}=C_{0},
$$

which accounts for the case $n=0$. Now, we suppose the case $n$ is satisfied, and set $v_{n}: B_{1} \rightarrow \mathbb{R}$ as

$$
v_{n}(x):=\frac{\left(u-P_{n}\right)\left(\rho^{n} x\right)}{\rho^{2 n}} .
$$

By the induction hypothesis and Lemma 3.2, there exists $h \in \mathcal{C}_{\text {loc }}^{2, \gamma}\left(B_{1}\right)$ satisfying

$$
\inf _{\beta \in \mathcal{B}}\left[-\operatorname{Tr}\left(\bar{A}_{\beta}(0)\left(D^{2} h+C_{n}\right)\right)\right]=\langle f\rangle \quad \text { in } \quad B_{8 / 9},
$$

such that

$$
\inf _{\beta \in \mathcal{B}}\left[-\operatorname{Tr}\left(\bar{A}_{\beta}(0) D^{2} h\right)\right]=0 \quad \text { in } \quad B_{8 / 9}
$$

and

$$
\left\|v_{n}-h\right\|_{L^{\infty}\left(B_{1 / 2}\right)} \leq \delta
$$

for any $\delta>0$ and $\gamma \in(0,1)$ being a universal constant. Now set

$$
\bar{P}_{n}(x):=h(0)+D h(0) \cdot x+\frac{1}{2} x^{T} D^{2} h(0) x .
$$

Then, by the triangular inequality and the regularity of $h$, we have

$$
\begin{aligned}
\sup _{B_{\rho}}\left|v_{n}-\bar{P}_{n}\right| & \leq\left\|v_{n}-h\right\|_{L^{\infty}\left(B_{1 / 2}\right)}+\sup _{B_{\rho}}\left|h-\bar{P}_{n}\right| \\
& \leq \delta+C \rho^{2} .
\end{aligned}
$$

Make the universal choices

$$
\delta=\frac{\rho^{2}}{2} \quad \text { and } \quad \rho=\left(\frac{1}{2 C}\right)^{1 / \gamma}
$$

to conclude

$$
\sup _{x \in B_{\rho}}\left|v_{n}(x)-\bar{P}_{n}(x)\right| \leq \rho^{2}
$$

Since

$$
\begin{aligned}
\sup _{B_{\rho}}\left|v_{n}-\bar{P}_{n}\right| & =\sup _{x \in B_{\rho}}\left|\frac{u\left(\rho^{n} x\right)-P_{n}\left(\rho^{n} x\right)}{\rho^{2 n}}-\bar{P}_{n}(x)\right| \\
& =\sup _{x \in B_{\rho^{n+1}}}\left|\frac{u(x)-P_{n}(x)-\rho^{2 n} \bar{P}_{n}\left(\rho^{-n} x\right)}{\rho^{2 n}}\right|,
\end{aligned}
$$

we have

$$
\sup _{x \in B_{\rho^{n+1}}}\left|u(x)-P_{n}(x)-\rho^{2 n} \bar{P}_{n}\left(\rho^{-n} x\right)\right| \leq \rho^{2 n} \rho^{2}=\rho^{2(n+1)} .
$$


Define

$$
\begin{aligned}
P_{n+1}(x) & :=P_{n}(x)-\rho^{2 n} \bar{P}_{n}\left(\rho^{-n} x\right) \\
& =P_{n}(x)+\rho^{2 n} h(0)+\rho^{n} D h(0) \cdot x+\frac{1}{2} x^{T} D^{2} h(0) x .
\end{aligned}
$$

Also, set

$$
a_{n+1}:=a_{n}+\rho^{2 n} h(0), \quad \mathbf{b}_{n+1}:=\mathbf{b}_{n}+\rho^{n} \operatorname{Dh}(0)
$$

and

$$
C_{n+1}:=C_{n}+D^{2} h(0)
$$

Notice that

$$
\inf _{\beta \in \mathcal{B}} \operatorname{Tr}\left(\bar{A}_{\beta}(0) C_{n+1}\right)=\inf _{\beta \in \mathcal{B}} \operatorname{Tr}\left(\bar{A}_{\beta}(0)\left(C_{n}+D^{2} h(0)\right)\right)=\langle f\rangle
$$

and there is $\widetilde{C}>0$ satisfying

$$
\begin{aligned}
\left|a_{n+1}-a_{n}\right| & +\rho^{n}\left\|\mathbf{b}_{n+1}-\mathbf{b}_{n}\right\|+\rho^{2 n}\left\|C_{n+1}-C_{n}\right\| \\
& =\rho^{2 n}|h(0)|+\rho^{2 n}\|D h(0)\|+\rho^{2 n}\left\|D^{2} h(0)\right\| \\
& \leq \widetilde{C} \rho^{2 n} .
\end{aligned}
$$

Hence, the statement is true in the case $n+1$, completing the induction argument.

The case $x_{0} \neq 0$ follows from a change of variables.

Theorem 3.4 (Estimates in $\mathcal{C}_{\text {loc }}^{1, \text { Log-Lip }}$ ) Let $u \in \mathcal{C}\left(B_{1}\right)$ be a viscosity solution to

$$
\sup _{\alpha \in \mathcal{A}} \inf _{\beta \in \mathcal{B}}\left[-\operatorname{Tr}\left(A_{\alpha, \beta}(x) D^{2} u\right)\right]=f(x) \quad \text { in } \quad B_{1} .
$$

Suppose the hypotheses of the Lemma 3.3 are in force and let $x_{0} \in B_{1 / 2}$. Then $u \in \mathcal{C}_{\text {loc }}^{1, \log -\operatorname{Lip}}\left(B_{1}\right)$ and there is a constant $C$ such that

$$
\sup _{x \in B_{r}\left(x_{0}\right)}\left|u(x)-u\left(x_{0}\right)-D u\left(x_{0}\right) \cdot\left(x-x_{0}\right)\right| \leq C\left(-r^{2} \ln (r)\right),
$$

for any $r \leq r_{0}$

Proof. We prove the case $x_{0}=0$. Taking

$$
P_{n}(x):=a_{n}+\mathbf{b}_{n} \cdot x+\frac{1}{2} x^{T} C_{n} x
$$

from Lemma 3.3, we have that

$$
\left|a_{n}-u(0)\right|=\left|P_{n}(0)-u(0)\right| \leq \rho^{2 n}
$$


and there exists a constant $C>0$ such that

$$
\begin{aligned}
\left|\mathbf{b}_{m}-\mathbf{b}_{n}\right| & \leq C \sum_{k=n+1}^{m}\left|\mathbf{b}_{k}-\mathbf{b}_{k-1}\right| \\
& \leq C \sum_{k=n+1}^{m} \rho^{k-1} \\
& =C \rho^{n} \cdot \frac{1-\rho^{m-n}}{1-\rho} \\
& \leq \widetilde{C} \rho^{n}
\end{aligned}
$$

for any $m>n$. Then $a_{n}$ and $\mathbf{b}_{n}$ are convergent because $0<\rho \ll 1$. We also have that

$$
\left\|C_{n}-C_{n-1}\right\| \leq C
$$

then

$$
\left\|C_{n}\right\| \leq\left\|C_{0}\right\|+\sum_{k=1}^{n}\left\|C_{k}-C_{k-1}\right\| \leq\left\|C_{0}\right\|+n C .
$$

Note that, for any $n \in \mathbb{N}$

$$
\begin{aligned}
& \left|u(x)-\left[u(0)+\mathbf{b}_{\infty} \cdot x\right]\right| \\
& \quad=\left|u(x)-P_{n}(x)+a_{n}+\mathbf{b}_{n} \cdot x+\frac{1}{2} x^{T} C_{n} x-\left[u(0)+\mathbf{b}_{\infty} \cdot x\right]\right| \\
& \quad \leq\left|u(x)-P_{n}(x)\right|+\left|a_{n}-u(0)\right|+\left|\left[\mathbf{b}_{n}-\mathbf{b}_{\infty}\right] \cdot x\right|+\frac{1}{2}\left\|x^{T} C_{n} x\right\| \\
& \quad \leq\left|u(x)-P_{n}(x)\right|+\left|a_{n}-u(0)\right|+\left\|\mathbf{b}_{n}-\mathbf{b}_{\infty}\right\| \cdot\|x\|+\frac{1}{2}\|x\|^{2}\left\|C_{n}\right\| \\
& \quad \leq\left|u(x)-P_{n}(x)\right|+\rho^{2 n}+C \rho^{n} \cdot\|x\|+\frac{1}{2}\|x\|^{2}\left(\left\|C_{0}\right\|+n C\right) .
\end{aligned}
$$

Where $b_{\infty}=\lim _{n \rightarrow \infty} b_{n}=D u(0)$ in fact. Then, for some $r \in(0,1 / 2)$

$$
\begin{aligned}
\sup _{x \in B_{r}(0)}|u(x)-[u(0)+D u(0) \cdot x]| \leq & \sup _{x \in B_{r}(0)}\left|u(x)-P_{n}(x)\right| \\
& +\rho^{2 n}+r C \rho^{n}+r^{2}\left\|C_{0}\right\|+n C r^{2} .
\end{aligned}
$$

Choosing $n$ such that $\rho^{n+1}<r \leq \rho^{n}$, then

$$
\sup _{x \in B_{r}(0)}\|u(x)-[u(0)+D u(0) \cdot x]\| \leq \rho^{2 n}+2 C \rho^{2 n}+\rho^{2 n}\left\|C_{0}\right\|+n C \rho^{2 n} .
$$

Since $\rho^{n+1}<r<1 / 2$ and $\rho^{-n} \leq r^{-1}$, we have

$$
\rho^{2 n}<\frac{r^{2}}{\rho^{2}}<\frac{r^{2} \ln \left(r^{-1}\right)}{\rho^{2} \ln (1 / 2)} \quad \text { and } \quad n \ln \left(\rho^{-1}\right) \leq \ln \left(r^{-1}\right)
$$


At the end we get

$$
\begin{aligned}
& \sup _{x \in B_{r}(0)} \mid u((x)-[u(0)+D h(0) \cdot x] \mid \\
&<\left(1+2 C+\left\|C_{0}\right\|\right) \frac{r^{2} \ln \left(r^{-1}\right)}{\rho^{2} \ln (1 / 2)}+\frac{C r^{2} \ln \left(r^{-1}\right)}{\rho^{2} \ln \left(\rho^{-1}\right)} \\
& \leq \widetilde{C} r^{2} \ln \left(r^{-1}\right),
\end{aligned}
$$

where

$$
\widetilde{C}=\max \left\{\frac{1+2 C+\left\|C_{0}\right\|}{\rho^{2} \ln (1 / 2)}, \frac{C}{\rho^{2} \ln \left(\rho^{-1}\right)}\right\} .
$$

The case $x_{0} \neq 0$ follows from a change of variables.

\section{3}

\section{Estimates in $\mathcal{C}_{\text {loc }}^{2, \gamma}\left(B_{1}\right)$}

Finally, we present the arguments concerning the regularity of the Isaacs equation in $\mathcal{C}^{2, \gamma}$. The result we detail branches in two variants; first, when the source term is a well-prepared function, we obtain $\mathcal{C}^{2, \gamma}$-regularity around the origin. Secondly, if $f \equiv 0$, we obtain $\mathcal{C}^{2, \gamma}$ - estimates locally in $B_{1}$. We first proof a lemma, which corresponds to the inductive step of the Lemma $\mathbf{3 . 5}$ (iteration result for $\mathcal{C}^{2, \gamma}$ regularity).

Lemma 3.5 Let $u \in \mathcal{C}\left(B_{1}\right)$ be a viscosity solution of

$$
\sup _{\alpha \in \mathcal{A}} \inf _{\beta \in \mathcal{B}}\left[-\operatorname{Tr}\left(A_{\alpha, \beta}(x) D^{2} u\right)\right]=f(x) \quad \text { in } \quad B_{1} \text {, }
$$

where $A_{\alpha, \beta}: B_{1} \times \mathcal{A} \times \mathcal{B} \rightarrow \mathbb{R}^{d^{2}}$ is a $(\lambda, \Lambda)$-elliptic matrix. Suppose that, for some $n \in \mathbb{N}$ there exist $\gamma \in(0,1), \rho \in(0,1 / 2)$ and a finite sequence of polynomials $\left(P_{k}\right)_{k \leq n}$, of the form

$$
P_{k}(x):=a_{k}+\mathbf{b}_{k} \cdot x+\frac{1}{2} x^{T} C_{k} x
$$

with $P_{0} \equiv P_{-1} \equiv 0$, satisfying

$$
\inf _{\beta \in \mathcal{B}}\left[-\operatorname{Tr}\left(\bar{A}_{\beta}(0) C_{k}\right)\right]=0 \quad \text { and } \quad\left\|u-P_{k}\right\|_{L^{\infty}\left(B_{\rho^{k}}\right)} \leq \rho^{k(2+\gamma)},
$$

for some $\bar{A}_{\beta}: B_{1} \times \mathcal{B} \rightarrow \mathbb{R}^{d^{2}}(\lambda, \Lambda)$-elliptic matrix. Moreover, suppose that for every $k \leq n$, we have

$$
\left|a_{k}-a_{k-1}\right|+\rho^{k-1}\left|\mathbf{b}_{k}-\mathbf{b}_{k-1}\right|+\rho^{2(k-1)}\left\|C_{k}-C_{k-1}\right\| \leq C \rho^{(k-1)(2+\gamma)} .
$$


Let $v_{n}: B_{1} \rightarrow \mathbb{R}$ be defined by

$$
v_{n}(x):=\frac{\left(u-P_{n}\right)\left(\rho^{n} x\right)}{\rho^{n(2+\gamma)}} .
$$

Then for every $\delta>0$, there exist $\epsilon_{3}>0$ and $h \in \mathcal{C}_{\text {loc }}^{2, \gamma}\left(B_{1}\right)$ solution of

$$
\begin{cases}\inf _{\beta \in \mathcal{B}}\left[-\operatorname{Tr}\left(\bar{A}_{\beta}(0)\left(D^{2} h+\frac{C_{n}}{\rho^{n \gamma}}\right)\right)\right]=0 & \text { in } \quad B_{8 / 9}, \\ h=v_{n} & \text { on } \quad \partial B_{8 / 9}\end{cases}
$$

such that, if

$$
f_{B_{r}}|f(x)|^{p} d x \leq \epsilon_{3}^{p} r^{\gamma p} \quad \text { and } \quad \sup _{x \in B_{r}}\left|A_{\alpha, \beta}(x)-\bar{A}_{\beta}(0)\right|<\epsilon_{3} r^{\gamma}
$$

uniformly in $\alpha$ and $\beta$, then

$$
\left\|v_{n}-h\right\|_{L^{\infty}\left(B_{7 / 8}\right)} \leq \delta
$$

for some $C>0$ universal constant.

Proof. We have that

$$
\begin{aligned}
D^{2} v_{n}(x) & =\frac{\rho^{2 n} D^{2} u\left(\rho^{n} x\right)-\rho^{2 n} C_{n}}{\rho^{n(2+\gamma)}} \\
& =\frac{D^{2} u\left(\rho^{n} x\right)-C_{n}}{\rho^{n \gamma}},
\end{aligned}
$$

and, for all $x \in B_{1}$,

$$
f\left(\rho^{n} x\right)=\sup _{\alpha \in \mathcal{A}} \inf _{\beta \in \mathcal{B}}\left[-\operatorname{Tr}\left(A_{\alpha, \beta}\left(\rho^{n} x\right) D^{2} u\left(\rho^{n} x\right)\right)\right]
$$

then

$$
\sup _{\alpha \in \mathcal{A}} \inf _{\beta \in \mathcal{B}}\left[-\operatorname{Tr}\left(A_{\alpha, \beta}\left(\rho^{n} x\right)\left(\rho^{n \gamma} D^{2} v_{n}(x)+C_{n}\right)\right)\right]=f\left(\rho^{n} x\right) .
$$

By taking

$$
f_{n}(x):=\frac{f\left(\rho^{n} x\right)}{\rho^{n \gamma}},
$$

we get that $v_{n}$ solves

$$
\sup _{\alpha \in \mathcal{A}} \inf _{\beta \in \mathcal{B}}\left[-\operatorname{Tr}\left(A_{\alpha, \beta}\left(\rho^{n} x\right)\left(D^{2} v_{n}+\frac{C_{n}}{\rho^{n \gamma}}\right)\right)\right]=f_{n}(x) \quad \text { in } \quad B_{1},
$$


where

$$
\begin{aligned}
\left\|f_{n}\right\|_{L^{p}\left(B_{1}\right)}^{p} & =\frac{1}{\rho^{n \gamma}} \int_{B_{1}}\left|f\left(\rho^{n} x\right)\right|^{p} d x \\
& =\frac{1}{\rho^{n \gamma}} \int_{B_{\rho^{n \gamma}}}|f(x)|^{p} \frac{d x}{\left|\rho^{n \gamma d}\right|} \\
& =\frac{1}{\rho^{n \gamma}} f_{B_{\rho^{n \gamma}}}|f(x)|^{p} d x \\
& \leq \frac{1}{\rho^{n \gamma}} \cdot \epsilon_{3}^{p} \rho^{n \gamma} \\
& =\epsilon_{3}^{p} .
\end{aligned}
$$

Finally, by Lemma 3.1, for any $\delta>0$ and some $\gamma \in(0,1)$, there exists $h \in \mathcal{C}_{\text {loc }}^{2, \gamma}\left(B_{1}\right)$ solving

$$
\begin{cases}\inf _{\beta \in \mathcal{B}}\left[-\operatorname{Tr}\left(\bar{A}_{\beta}(0)\left(D^{2} h+\frac{C_{n}}{\rho^{n \gamma}}\right)\right)\right]=0 & \text { in } \quad B_{8 / 9}, \\ h=v_{n} & \text { on } \quad \partial B_{8 / 9},\end{cases}
$$

such that

$$
\left\|v_{n}-h\right\|_{L^{\infty}\left(B_{1 / 2}\right)} \leq \delta
$$

Lemma 3.6 Let $u \in \mathcal{C}\left(B_{1}\right)$ be a viscosity solution of

$$
\sup _{\alpha \in \mathcal{A}} \inf _{\beta \in \mathcal{B}}\left[-\operatorname{Tr}\left(A_{\alpha, \beta}(x) D^{2} u\right)\right]=f(x) \quad \text { in } \quad B_{1}
$$

where $A_{\alpha, \beta}: B_{1} \times \mathcal{A} \times \mathcal{B} \rightarrow \mathbb{R}^{d^{2}}$ is a $(\lambda, \Lambda)$-elliptic matrix and $\|u\|_{\mathcal{C}\left(\bar{B}_{1}\right)} \leq 1$. Then there exist $\gamma \in(0,1), C>0, \epsilon_{3}>0, \rho \in(0,1 / 2)$ and a sequence of polynomials $\left(P_{n}\right)_{n \in \mathbb{N}}$, given by

$$
P_{n}(x):=a_{n}+\mathbf{b}_{n} \cdot x+\frac{1}{2} x^{T} C_{n} x,
$$

such that, if

$$
\sup _{x \in B_{r}}\left|A_{\alpha, \beta}(x)-\bar{A}_{\beta}\left(x_{0}\right)\right|<\epsilon_{3} r^{\gamma} \quad \text { and } \quad f_{B_{r}}|f(x)|^{p} d x \leq \epsilon_{3}^{p} r^{\gamma p} \text {, }
$$

for every $x_{0} \in B_{1}$ and (alpha, $\left.\beta\right) \in \mathcal{A} \times \mathcal{B}$, then

$$
\inf _{\beta \in \mathcal{B}}\left[-\operatorname{Tr}\left(\bar{A}_{\beta}\left(x_{0}\right) C_{n}\right)\right]=0, \quad\left\|u-P_{n}\right\|_{L^{\infty}\left(B_{\rho^{n}}\right)} \leq \rho^{n(2+\gamma)} .
$$

and

$$
\left|a_{n}-a_{n-1}\right|+\rho^{n-1}\left|\mathbf{b}_{n}-\mathbf{b}_{n-1}\right|+\rho^{2(n-1)}\left\|C_{n}-C_{n-1}\right\| \leq C \rho^{(n-1)(2+\gamma)},
$$


for any $n \geq 0$.

Proof. We proceed by induction in $n \in \mathbb{N}$ and consider the case $x_{0}=0$. In the basic case, take $P_{-1} \equiv P_{0} \equiv 0$. Hence

$$
a_{0}=a_{-1}=0 \quad, \mathbf{b}_{0}=\mathbf{b}_{-1}=0 \quad \text { and } \quad C_{0}=C_{-1}=0,
$$

which satisfy the conditions for $n=0$.

Now, we suppose the case $n$ is satisfied, and let $v_{n}: B_{1} \rightarrow \mathbb{R}$ be defined by

$$
v_{n}(x):=\frac{\left(u-P_{n}\right)\left(\rho^{n} x\right)}{\rho^{n(2+\gamma)}} .
$$

By the induction hypotheses and Lemma 3.5, for every $\delta>0$, there exists $h \in \mathcal{C}_{\text {loc }}^{2, \gamma}\left(B_{1}\right)$ satisfying

$$
\inf _{\beta \in \mathcal{B}}\left[-\operatorname{Tr}\left(\bar{A}_{\beta}(0)\left(D^{2} h+\frac{C_{n}}{\rho^{n \gamma}}\right)\right)\right]=0,
$$

such that

$$
\left\|v_{n}-h\right\|_{L^{\infty}\left(B_{7 / 8}\right)} \leq \delta
$$

Set

$$
\bar{P}_{n}(x):=h(0)+D h(0) \cdot x+\frac{1}{2} x^{T} D^{2} h(0) x .
$$

Then, by the triangular inequality and the regularity of $h$, we have

$$
\sup _{B_{\rho}}\left|v_{n}-\bar{P}_{n}\right| \leq\left\|v_{n}-h\right\|_{L^{\infty}\left(B_{1 / 2}\right)}+\sup _{B_{\rho}}\left|h-\bar{P}_{n}\right| \leq \delta+C \rho^{2+\gamma} .
$$

Choose

$$
\delta=\frac{\rho^{2+\gamma}}{2} \text { and } \rho=\left(\frac{1}{2 C}\right)^{\frac{1}{\gamma-\gamma}}
$$

to ensure

$$
\sup _{B_{\rho}}\left|v_{n}(x)-\bar{P}_{n}(x)\right| \leq \rho^{2+\gamma} .
$$

Hence,

$$
\begin{aligned}
\sup _{x \in B_{\rho^{n+1}}}\left|u(x)-P_{n}(x)-\rho^{n(2+\gamma)} \bar{P}_{n}\left(\rho^{-n} x\right)\right| \\
=\sup _{x \in B_{\rho}}\left|u\left(\rho^{n} x\right)-P_{n}\left(\rho^{n} x\right)-\rho^{n(2+\gamma)} \bar{P}_{n}(x)\right| \\
=\rho^{n(2+\gamma)} \sup _{B_{\rho}}\left|v_{n}(x)-\bar{P}_{n}(x)\right| \\
\leq \rho^{n(2+\gamma)} \rho^{2+\gamma}=\rho^{(n+1)(2+\gamma)} .
\end{aligned}
$$


Define

$$
P_{n+1}(x):=P_{n}(x)-\rho^{n(2+\gamma)} \bar{P}_{n}\left(\rho^{-n} x\right)
$$

Also, set

$$
a_{n+1}=a_{n}+\rho^{n(2+\gamma)} h(0), \quad \mathbf{b}_{n+1}=\mathbf{b}_{n}+\rho^{n(1+\gamma)} \operatorname{Dh}(0)
$$

and

$$
C_{n+1}=C_{n}+\rho^{n \gamma} D^{2} h(0)
$$

We finally have

$$
\inf _{\beta \in \mathcal{B}}\left[-\operatorname{Tr}\left(\bar{A}_{\beta}(0) C_{n+1}\right)\right]=\rho^{n \gamma} \inf _{\beta \in \mathcal{B}}\left[-\operatorname{Tr}\left(\bar{A}_{\beta}(0)\left(D^{2} h+\frac{C_{n}}{\rho^{n \gamma}}\right)\right)\right]=0,
$$

and there is $\widetilde{C}>0$ satisfying

$$
\left|a_{n+1}-a_{n}\right|+\rho^{n}\left\|\mathbf{b}_{n+1}-\mathbf{b}_{n}\right\|+\rho^{2 n}\left\|C_{n+1}-C_{n}\right\| \leq \widetilde{C} \rho^{n(2+\gamma)} .
$$

Hence, the statement is true in the case $n+1$, completing the induction argument. The case $x_{0} \neq 0$ follows from a change of variables.

Theorem 3.7 (Estimates in $\mathcal{C}^{2, \gamma}$ ) Let $u \in \mathcal{C}\left(B_{1}\right)$ be a viscosity solution to

$$
\sup _{\alpha \in \mathcal{A}} \inf _{\beta \in \mathcal{B}}\left[-\operatorname{Tr}\left(A_{\alpha, \beta}(x) D^{2} u\right)\right]=f(x) \quad \text { in } \quad B_{1} .
$$

Suppose the hypotheses of the Lemma $\mathbf{3 . 5}$ are in force. Then there exists $\gamma \in(0,1)$ such that $u$ is of class $\mathcal{C}^{2, \gamma}$ at the origin. If $f \equiv 0$, we have $u \in \mathcal{C}_{\text {loc }}^{2, \gamma}\left(B_{1}\right)$ and there exists a universal constant $C>0$ such that

$$
\|u\|_{\mathcal{C}^{2, \gamma}\left(B_{1 / 2}\right)} \leq C\|u\|_{L^{\infty}\left(B_{1}\right)}
$$

Proof. By Lemma 3.6, there are $\gamma \in(0,1), \rho \in(0,1 / 2)$ and a sequence of polynomials $\left(P_{n}\right)_{n \in \mathbb{N}}$, of the form

$$
P_{n}(x):=a_{n}+\mathbf{b}_{n} \cdot x+\frac{1}{2} x^{T} C_{n} x \quad, P_{0} \equiv P_{-1} \equiv 0
$$

satisfying

$$
\left\|u-P_{n}\right\|_{L^{\infty}\left(B_{\rho^{n}}\right)} \leq \rho^{n(2+\gamma)}
$$

with

$$
\left|a_{n}-a_{n-1}\right|+\rho^{n-1}\left|\mathbf{b}_{n}-\mathbf{b}_{n-1}\right|+\rho^{2(n-1)}\left\|C_{n}-C_{n-1}\right\| \leq C \rho^{(n-1)(2+\gamma)}
$$


for every $n \geq 0$. Hence

$$
\left|a_{n}-u(0)\right|=\left|P_{n}(0)-u(0)\right| \leq \rho^{n(2+\gamma)},
$$

and

$$
\left|\mathbf{b}_{m}-\mathbf{b}_{n}\right| \leq C \sum_{k=n+1}^{m}\left|\mathbf{b}_{k}-\mathbf{b}_{k-1}\right| \leq C \sum_{k=n+1}^{m} \rho^{(k-1) \gamma} \leq \frac{C}{1-\rho^{\gamma}} \cdot \rho^{n \gamma}
$$

for any $m>n$. Then $a_{n}$ and $\mathbf{b}_{n}$ are convergent because $0<\rho \ll 1$, thus they are bounded. . We also have that

$$
\left\|C_{k}-C_{k-1}\right\| \leq C \rho^{(k-1) \gamma}
$$

which implies

$$
\begin{aligned}
\left\|C_{n}\right\| & \leq\left\|C_{0}\right\|+\sum_{k=1}^{n}\left\|C_{k}-C_{k-1}\right\| \\
& \leq\left\|C_{0}\right\|+C \sum_{k=1}^{n} \rho^{(k-1) \gamma} \\
& =\left\|C_{0}\right\|+C \frac{1-\rho^{n \gamma}}{1-\rho^{\gamma}} \\
& <\left\|C_{0}\right\|+\frac{C}{1-\rho^{\gamma}}=\widetilde{C}<\infty .
\end{aligned}
$$

Thus, we conclude that $u \in \mathcal{C}_{\text {loc }}^{2, \gamma}\left(B_{1}\right)$.

Finally, the inequality (3-1) follows from the interior Hölder regularity (Theorem 1.10 and [(CC95), Propositions 4.12 and 4.13]). 


\section{Bibliography}

[C89] CAFFARELLI, L. A.. Interior a priori estimates for solutions of fully non-linear equations. Annals of Mathematics, 130(1):189-213, 1989.

[CC95] CAFFARELLI, L.; CABRÉ, X.. Fully nonlinear elliptic equations, volumen 43 de American Mathematical Society Colloquium Publications. American Mathematical Society, Providence, RI, 1995.

[CCS96] CAFFARELLI, L.; KOCAN, M.; CRANDALL, M. ; ŚWIẸCH, A.. On viscosity solutions of fully nonlinear equations with measurable ingredients. Communications on Pure and Applied Mathematics, 49(4):365397, 1996.

[E10] EVANS, L.. Partial Differential Equations, volumen 19 de Graduate Studies in Mathematics. American Mathematical Society, Providence, Ri, second edition, 2010 .

[HL11] HAN, Q.; LIN, F.-H.. Elliptic partial differential equations. Courant lecture notes. Courant Institute of Mathematical Sciences, Robotics Lab, New York University, 2nd ed. edition, 2011. Includes bibliographical references ( $p$. 147).

[165] ISAACS, R.. Differential games: a mathematical theory with applications to warfare and pursuit, control and optimization. John Wiley \& Sons, Inc., New York-London-Sydney, 1965.

[LZ15] LI, D.; ZHANG, K.. W2,p interior estimates of fully nonlinear elliptic equations. Bulletin of the London Mathematical Society, 47(2):301314, 2015.

[P19] PIMENTEL, E. A.. Regularity theory for the isaacs equation through approximation methods. Annales de l'Institut Henri Poincaré C, Analyse non linéaire, 36(1):53 - 74, 2019.

[S97] ŚWIĘCH, A.. W1,p-interior estimates for solutions of fully nonlinear, uniformly elliptic equations. Advances in Differential Equations, 2(6):1005-1027, 1997. 